\title{
Dual-polarimetric descriptors from Sentinel-1 GRD SAR data for crop growth assessment
}

\author{
Narayanarao Bhogapurapu ${ }^{\mathrm{a}, *}$, Subhadip Dey ${ }^{\mathrm{a}}$, Avik Bhattacharya ${ }^{\mathrm{a}}$, \\ Dipankar Mandal ${ }^{\mathrm{a}}$, Juan M. Lopez-Sanchez ${ }^{\mathrm{b}}$, Heather McNairn ${ }^{\mathrm{c}}$, \\ Carlos López-Martínez ${ }^{\mathrm{d}}$, Y. S. Rao ${ }^{\mathrm{a}}$ \\ ${ }^{a}$ Microwave Remote Sensing Lab, Centre of Studies in Resources Engineering, \\ Indian Institute of Technology Bombay, Mumbai, India \\ ${ }^{b}$ University of Alicante, Alicante, Spain \\ ${ }^{c}$ Ottawa Research and Development Centre, Agriculture and Agri-Food Canada, Canada \\ ${ }^{d}$ Signal Theory and Communications Department (TSC), Universitat Politécnica de \\ Catalunya $(U P C)$, Barcelona, Spain
}

\begin{abstract}
Accurate and high-resolution spatio-temporal information about crop phenology obtained from Synthetic Aperture Radar (SAR) data is an essential component for crop management and yield estimation at a local scale. Crop growth monitoring studies seldom exploit complete polarimetric information contained in dual-pol GRD SAR data. In this study, we propose three polarimetric descriptors: the pseudo scattering-type parameter $\left(\theta_{c}\right)$, the pseudo scattering entropy parameter $\left(H_{c}\right)$, and the co-pol purity parameter $\left(m_{c}\right)$ from dual-pol S1 GRD SAR data. We also introduce a novel unsupervised clustering framework using $H_{c}$ and $\theta_{c}$ with six clustering zones to represent various scattering mechanisms. We implemented the proposed algorithm on the cloud-based Google Earth Engine (GEE) platform for Sentinel-1 SAR data. We have shown the sensitivity of these descriptors over a time series of
\end{abstract}

\footnotetext{
*Corresponding author: N. Bhogapurapu (narayanarao.bhogapurapu@gmail.com)
} 
data for wheat and canola crops at a test site in Canada. From the leaf development stage to the flowering stage for both crops, the pseudo scattering-type parameter $\theta_{c}$ changes by approximately $17^{\circ}$. Moreover, within the entire phenology window, both $m_{c}$ and $H_{c}$ varies by about 0.6 . The effectiveness of $\theta_{c}$ and $H_{c}$ to cluster the phenological stages for the two crops is also evident from the clustering plot. During the leaf development stage, about $90 \%$ of the sampling points were clustered into the low to medium entropy scattering zone for both the crops. Throughout the flowering stage, the entire cluster shifted into the high entropy vegetation scattering zone. Finally, during the ripening stage, the clusters of sample points were split between the high entropy vegetation scattering zone and the high entropy distributed scattering zone, with $>55 \%$ of the sampling points in the high entropy distributed scattering zone. This innovative clustering framework will facilitate the operational use of S1 GRD SAR data for agricultural applications. Keywords: GRD SAR, Dual-pol, phenology, Unsupervised clustering, GEE, Sentinel-1

\section{1. Introduction} crop growth monitoring and classification, yield estimation, and phenolog4 ical stages characterization. This is due to their high sensitivity towards 5 the structure and dielectric properties of crop canopies Ulaby, 1975; Ulaby 6 and El-Rayes, 1987; Brisco et al., 1992; Ferrazzoli et al., 1992; McNairn and 7 Brisco, 2004; Steele-Dunne et al., 2017). Because of its high spatial reso-

8 lution and all-weather capabilities, SAR has proven to be a promising data 
source for continuously monitoring crops at field scales. The interaction of the SAR signal with crop canopies and the underlying soil varies with wavelength, polarization and angle of incidence (Ferrazzoli et al., 1992; Davidson et al., 2000). In general, during the early vegetative growth stage, the SAR backscatter signal is significantly affected by the underlying soil (Wiseman et al., 2014). The canopy structure and canopy moisture distribution are among major observable biophysical parameters that influence backscatter at each phenological stage. Further, the dense and complex geometry of the canopy leads to randomness in the scattering, which is more significant for fully developed crop canopies Mascolo et al., 2016; Hariharan et al., 2018; Wang et al., 2019). The scattering becomes increasingly unpredictable during fruit development stages, leading to greater randomness in the SAR response (Jiao et al. 2014).

The availability of dual-pol SAR data acquired by the Sentinel-1 constellation provides diverse opportunities for many crop monitoring applications (ESA, 2017). Compared to full-pol mode, dual-pol modes have advantages in terms of larger swath widths and lower data volumes, but at the expense of reduced polarimetric information (Lee et al., 2001; Ainsworth et al., 2009). The Sentinel-1 (S1) SAR sensor in Interferometric Wide (IW) swath mode acquires data in dual-polarization, either in VV-VH or HH-HV.

Several researchers indicated the potential use of dual-pol backscatter intensities for crop type identification (Kussul et al., 2016, Nguyen et al., 2016; Bargiel, 2017; Van Tricht et al., 2018; Mandal et al., 2018; Whelen and Siqueira, 2018; Minasny et al., 2019; Arias et al., 2020), crop biophysical parameter estimation Bousbih et al., 2017; Kumar et al., 2018; Mandal 
et al. 2020a), and phenology identification (Nelson et al., 2014, De Bernardis et al., 2015; Lasko et al., 2018; Singha et al., 2019; Fikriyah et al., 2019). Cloude (2007) proposed a clustering technique for dual-polarimetric (HH-HV or $\mathrm{VV}-\mathrm{VH}) \mathrm{SAR}$ data. An eigendecomposition of the $2 \times 2$ covariance matrix is performed to characterize scattering mechanisms from targets. The average scattering angle $\bar{\alpha}$ is obtained from the two orthogonal polarization states weighted by their corresponding pseudo probabilities obtained from the eigenvalues. The entropy $H$ is obtained from the pseudo probabilities. Ainsworth et al. (2008) introduced a scattering-type parameter $\theta$ for dual-pol SLC data (HH-HV) utilizing the eigendecomposition technique. This parameter is presented as a measure between the cross- and co-pol backscatter ratio $\left(\sigma_{\mathrm{XY}}^{\circ} / \sigma_{\mathrm{XX}}^{\circ}\right)$. It was stated that although the formulation is similar to Cloude $\alpha$, the scattering information content is different. Utilizing $\theta$ and the scattering entropy $(H)$ for dual-pol SAR data, an unsupervised clustering framework was proposed to identify different targets based on their scattering mechanisms. The unsupervised clustering plane was divided into eight different zones based on the scattering types.

Besides this, several vegetation descriptors such as the Radar Vegetation Index (RVI) for dual-pol (Trudel et al., 2012), Dual-Pol SAR Vegetation Index (DPSVI) (Periasamy, 2018), and Dual-pol Radar Vegetation Index (DpRVI) (Mandal et al., 2020b) have been developed for crop growth monitoring and biophysical parameter retrieval. However, similar descriptors are not directly available for dual-pol GRD SAR data products.

In particular, investigation often is limited to the direct use of backscatter intensities or their ratios for crop phenology identification and cluster- 
ing. Vreugdenhil et al. (2018) studied the sensitivity of backscatter intensities and the cross-pol ratio (VH/VV) to crop biophysical parameters such as the Vegetation Water Content (VWC), Leaf Area Index (LAI), biomass, and the plant height for three different crops using the Sentinel-1 GRD SAR data.

Temporal sensitivity analysis using various machine learning models has shown that the cross-pol ratio is a valuable parameter for monitoring crop biophysical parameters and phenology. Song and Wang (2019) analyzed the temporal trend of $\mathrm{VV}$ and $\mathrm{VH}$ backscatter intensities to identify and map winter wheat crop using a parallelepiped classifier. They distinguished different phenology stages by exploring the temporal trend of the VH/VV ratio and its slope.

Nasrallah et al. (2019) fitted multiple Gaussian functions to a timeseries of backscatter intensities ( $\mathrm{VV}, \mathrm{VH}$ and $\mathrm{VH} / \mathrm{VV}$ ) to estimate the date of significant phenology stages for wheat. Wali et al. (2020) explored the sensitivity of temporal backscatter intensities of rice biophysical parameters using a combination of linear regression lines. With this approach, they were able to identify the reproductive growth stages of rice. Schlund and Erasmi (2020) demonstrated the sensitivity of interferometric phase information to estimate the dates of different phenology stages of wheat.

Information about phenological status can increase crop classification accuracy (Bargiel, 2017; Li et al., 2019). However, available studies on crop monitoring using GRD SAR data are mostly limited to the direct use of backscatter intensities and their ratios, along with a few empirical models. These approaches partly utilize the available polarimetric information from dual-pol GRD SAR data. Dual-polarimetric descriptors that characterize 
different target scattering mechanisms have a wide range of applicability compared to empirical and data-driven models limited to specific crops and regions. In this regard, an unsupervised clustering framework that suitably utilizes the available polarimetric information from dual-pol GRD SAR data is needed to monitor crop growth dynamics.

In general, polarimetric parameters have been directly attributed to the physical properties of the crop canopy (Lopez-Sanchez et al., 2012, 2014; McNairn et al., 2018; Dey et al., 2020b), and has therefore helped monitor crop phenology. Unfortunately, the polarimetric parameters reported in these studies are not immediately apparent in the case of dual-pol GRD SAR data. The majority of SAR-based crop monitoring studies were limited to small study areas due to the high volume of data processing. For example, the Sentinel-1 constellation acquires data at a rate of approximately 600 GB per day (Ali et al. 2017). This volume of data requires high storage and computational resources for processing. Unfortunately, these resources are limited and restricted for full exploitation to those with access to High-Performance Computing Systems (HPCS). With the advent of cloud platforms such as the Google Earth Engine (GEE) (Gorelick et al., 2017), the NASA Earth Exchange (Nemani et al. 2011), Amazon Web Services (AWS) (Jackson et al., 2010), and Microsoft Azure (Redkar et al., 2009), large-scale remote sensing and geospatial data analysis have become possible with minimum local computational resources (Hird et al., 2017). In this aspect, the web-based GEE platform is designed to make planetary-scale remote sensing data processing manageable and efficient (Gorelick et al., 2017). The free-to-use policy and various in-built GEE algorithms make it an ideal tool for both experts 
and non-experts alike. The major contributions of the current study are as follows:

- Introduces three new dual-polarimetric descriptors: $m_{c}, \theta_{c}$, and $H_{c}$.

- Proposes a new unsupervised clustering framework using two parameters $\left(\theta_{c}\right.$ and $\left.H_{c}\right)$ obtained from the dual-pol GRD SAR data.

- Six feasible clustering zones depicting different scattering mechanisms.

- Specific to crop monitoring, the proposed clustering framework effectively characterizes different phenological stages.

- Demonstrates how the proposed algorithm is implemented on GEE, making it available for global monitoring with minimal local computational requirements.

The performance of the parameters and clustering framework is analyzed using time-series Sentinel-1 SAR data for monitoring wheat and canola.

\section{Study area and dataset}

The test site is located near Carman, Manitoba (Canada), covering an intensively cropped area of $26 \mathrm{~km} \times 48 \mathrm{~km}$. The dominant crops grown in this region include wheat, canola, soybeans, corn and oats, along with a small fraction of acreages in grassland and pasture. The sowing period of crops in this region varies from early to late May, depending on crop variety and cultivation practices. The harvesting period extends until late September. 
The nominal size of each field is approximately $800 \mathrm{~m} \times 800 \mathrm{~m}$. Each field comprises 16 sampling locations arranged in two parallel transects separated by $200 \mathrm{~m}$, as shown in Figure 1. During the SMAPVEX-16 campaign, in-situ measurement of vegetation and soil was collected for 50 fields near coincident with satellite acquisitions.

In this study, we have considered 24 fields (13 wheat and 11 canola) for analysis. Figure 1 presents the distribution of the selected fields in the study area. One can find additional details regarding in-situ sampling methods during the SMAPVEX-16 campaign in McNairn et al. (2016); Bhuiyan et al. (2018).

Figures 2 and 3 provide field photos of different growth stages of wheat and canola, respectively. The Manitoba weekly agriculture reports Agriculture (2016) provide additional details regarding crop conditions. Sentinel1 operates at C-band with a central transmit frequency of $5.405 \mathrm{GHz}$. In this work, we have utilized the data acquired with the Interferometric Wide swath (IW) mode with a swath width of $250 \mathrm{~km}$. The spatial resolution is $5 \mathrm{~m} \times 20 \mathrm{~m}$ in range and azimuth, respectively, and the Noise Equivalent Sigma Zero (NESZ) is $-25 \mathrm{~dB}$ with the incidence angle varying between $20^{\circ}$ to $46^{\circ}$. From the available Sentinel-1 images acquired during the campaign, we have used eight dual-pol (VV-VH) C-band Sentinel-1A GRD SAR in the present study. We have utilized the VV-VH data acquired with IW mode with incidence angle ranging from $30.65^{\circ}$ to $41.76^{\circ}$. Complete details of the SAR data utilized in the study are presented in Table 1.

The data were chosen based on the availability of in-situ measurements of crop phenology stages and coincident Sentinel-1A acquisitions for six days 

(DOY-146, 165, 182, 189, 206, 225).
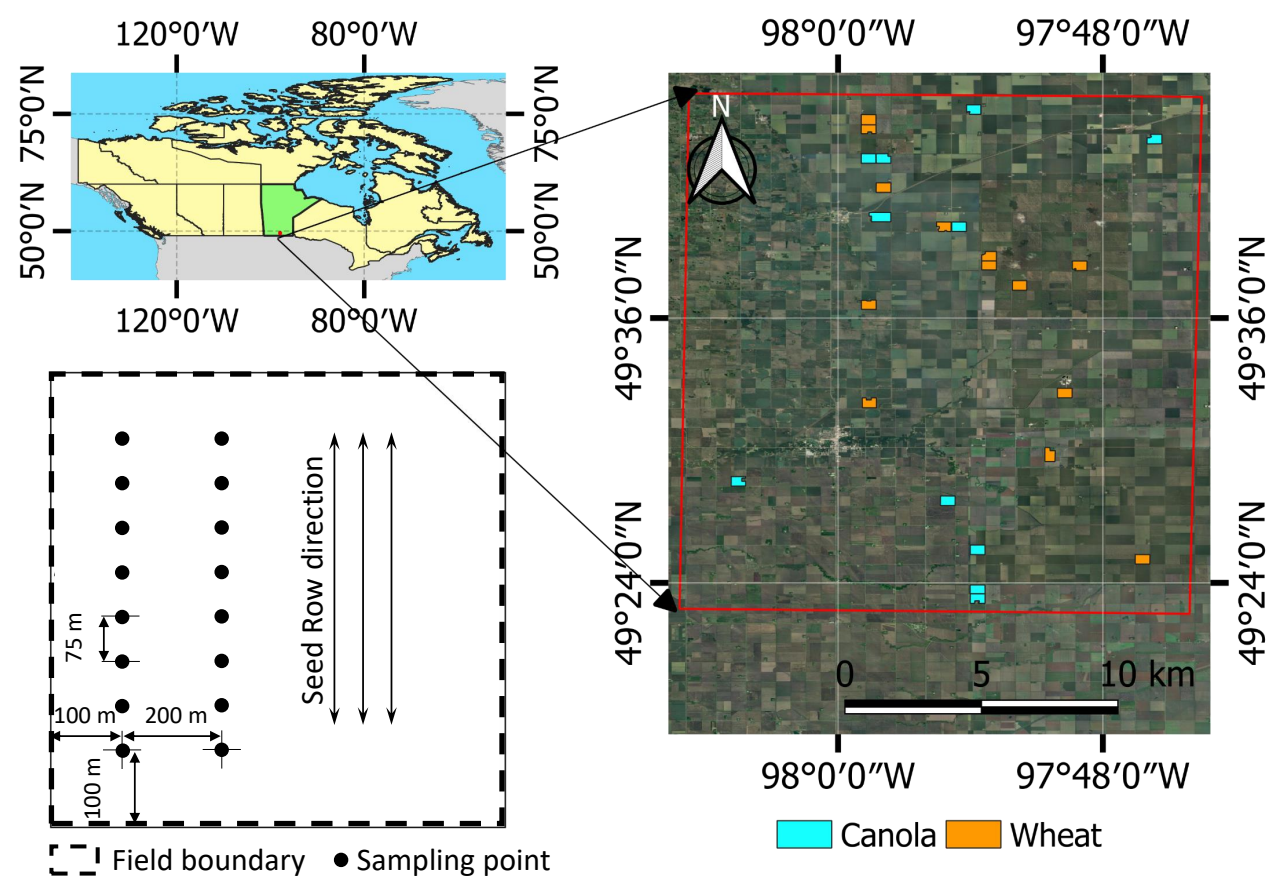

of the year (DOY) for wheat (DOY-146, 165, 182, 189, 201, 230) and canola

Figure 1: The study area and the distribution of wheat and canola fields in the study area overlaid on a Google earth image. The sampling schema followed for ground truth collection is detailed in the dashed rectangle (bottom left).

\section{Methodology}

This section proposes three descriptors from the Level-1 S1 GRD SAR data. We express the co-pol purity parameter in terms of the co-pol to cross-pol ratio, which is then used to obtain the scattering-type parameter.

The measure of scattering randomness is expressed in terms of the ratio parameter. We utilize these descriptors to introduce a clustering framework 
Table 1: Details and specification of Sentinel-1A data used in the present study. Data are acquired from the Carman test site during the SMAPVEX16-MB campaign. The range of incidence angles shown is specific to the location of the sample sites (IW: Interferometric Wide swath)

\begin{tabular}{ccccc}
\hline Date & DOY & $\begin{array}{c}\text { Acquisition } \\
\text { Mode }\end{array}$ & $\begin{array}{c}\text { Incidence angle } \\
\text { range (deg.) }\end{array}$ & Orbit \\
\hline 25-May-16 & 146 & IW & $40.18-41.76$ & Ascending \\
13-Jun-16 & 165 & IW & $30.65-32.70$ & Ascending \\
30-Jun-16 & 182 & IW & $40.17-41.75$ & Ascending \\
07-Jul-16 & 189 & IW & $30.64-32.69$ & Ascending \\
19-Jul-16 & 201 & IW & $30.70-32.70$ & Ascending \\
24-Jul-16 & 206 & IW & $40.16-41.74$ & Ascending \\
12-Aug-16 & 225 & IW & $30.65-32.70$ & Ascending \\
17-Aug-16 & 230 & IW & $40.16-41.74$ & Ascending \\
\hline
\end{tabular}

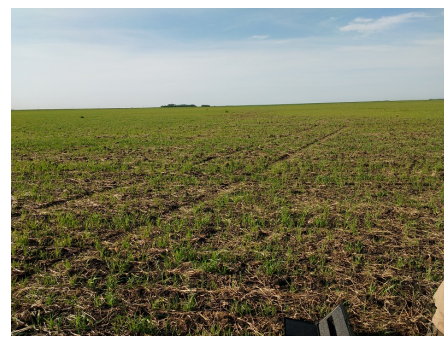

(a) Leaf Development - Tillering

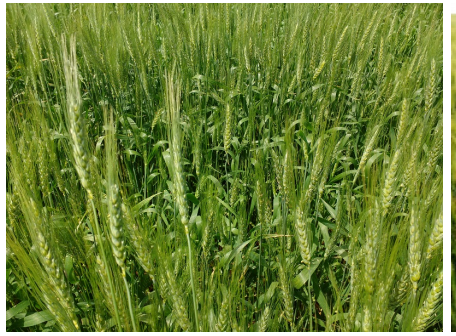

(d) Flowering-Fruit Development

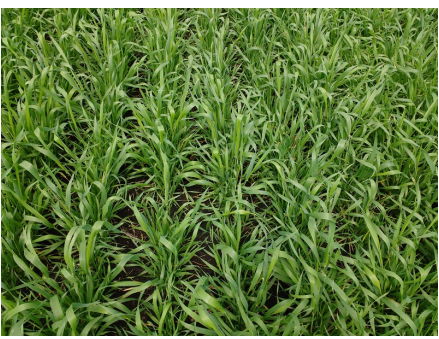

(b) Stem Elongation-Booting

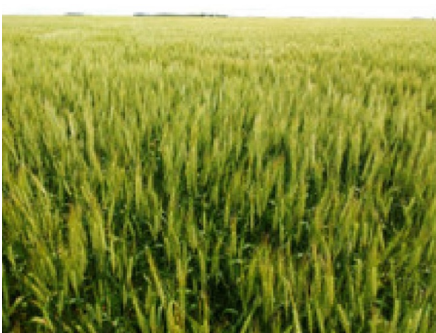

(e) Dough formation

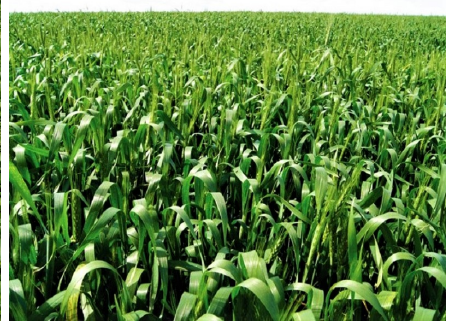

(c) Heading

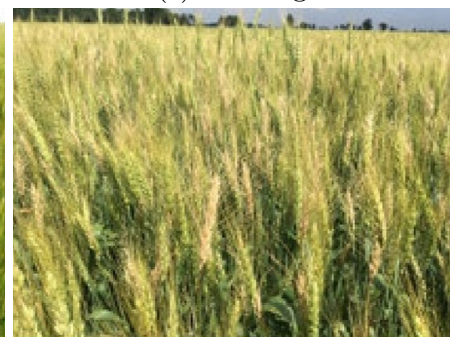

(f) Maturity

Figure 2: Field photos showing different phenology stages of wheat.

for crop phenology identification. Finally, we present the overall processing chain of the framework using the GEE platform. 


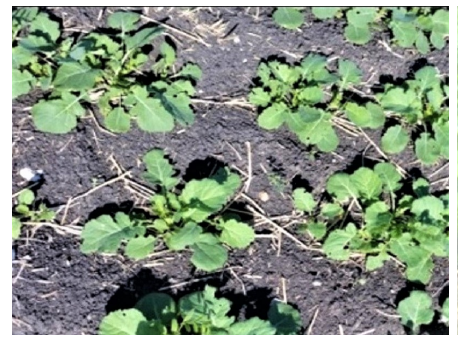

(a) Leaf Development - Side shoot formation

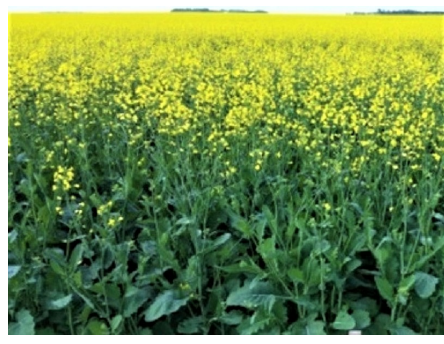

(d) Flowering

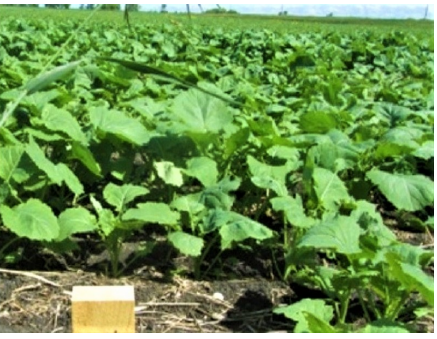

(b) Stem Elongation

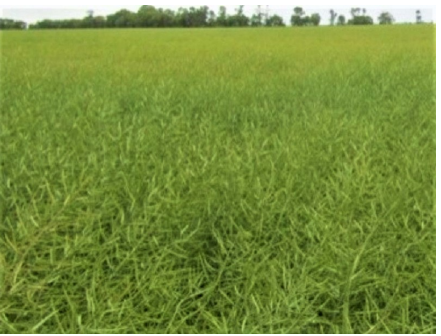

(e) Pod Development

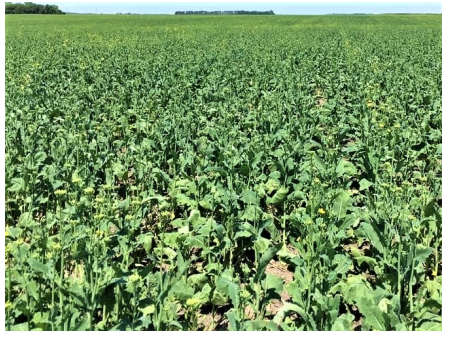

(c) Inflorescence Emergence

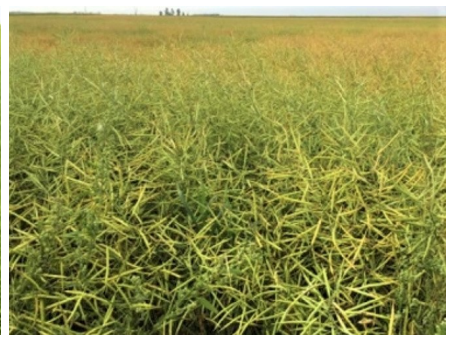

(f) Ripening

Figure 3: Field photos showing different phenology stages of canola.

\subsection{Dual-polarimetric descriptors}

In this section, we present three dual-polarimetric descriptors from the Level-1 S1 GRD SAR data. We propose an unsupervised clustering framework to monitor different crop growth stages based on their diverse scattering characteristics using these descriptors. In the Level-1 S1 GRD SAR data product, we obtain backscatter response either in $\left(\sigma_{\mathrm{VV}}^{\circ}, \sigma_{\mathrm{VH}}^{\circ}\right)_{\mathrm{dB}}$ or $\left(\sigma_{\mathrm{HH}}^{\circ}, \sigma_{\mathrm{HV}}^{\circ}\right)_{\mathrm{dB}}$ modes, where $\mathrm{H}$ and $\mathrm{V}$ are respectively the horizontal and vertical transmit and receive polarization components. The subscript $\mathrm{dB}$ represents the GRD SAR data products in decibel $(\mathrm{dB})$ scale. In general, for a monostatic antenna configuration and a natural scene, we assume $\sigma_{\mathrm{XY}}^{\circ} \leq \sigma_{\mathrm{XX}}^{\circ}$ (where $\mathrm{X}$ and $\mathrm{Y}$ are $\mathrm{H}$ or $\mathrm{V}$ polarizations respectively) (Cloude, 2009). Using this assumption, we consider the ratio parameter, $0 \leq q=\frac{\sigma_{\mathrm{XY}}^{\circ}}{\sigma_{\mathrm{XX}}^{\circ}} \leq 1$, in the linear scale. This parameter has been widely used in the literature as a descriptor for 
several crop monitoring applications (Della Vecchia et al., 2008; Vreugdenhil et al., 2018; Homayouni et al., 2019). In the GRD product, we do not keep the relative phase information between the XX and XY polarization. Hence, we cannot obtain covariance information from the GRD product, unlike the SLC data. We express the co-pol purity parameter $\left(m_{c}\right)$ in terms of $q$ given in Equation 1. It can be noted that for $q=1, m_{c}=0$, and for $q=0, m_{c}=1$. In between these two extreme cases, $1>q>0,0<m_{c}<1$.

$$
m_{c}=\frac{1-q}{1+q} ; \quad 0 \leq m_{c} \leq 1
$$

Utilizing $\sigma_{\mathrm{XX}}^{\circ}, \sigma_{\mathrm{XY}}^{\circ}$, and $m_{c}$ we define two auxiliary quantities as,

$$
\tan \zeta_{1}=\frac{\sigma_{\mathrm{XX}}^{\circ}}{m_{c} I} \quad \text { and } \quad \tan \zeta_{2}=\frac{\sigma_{\mathrm{XY}}^{\circ}}{m_{c} I},
$$

where the total intensity, $I=\sigma_{\mathrm{XX}}^{\circ}+\sigma_{\mathrm{XY}}^{\circ}$. By using a simple relationship, we obtain,

$$
\begin{aligned}
\tan \theta_{c} & =\tan \left(\zeta_{1}-\zeta_{2}\right) \\
& =\frac{(1-q)^{2}}{1+q^{2}-q} ; \quad 0^{\circ} \leq \theta_{c} \leq 45^{\circ}
\end{aligned}
$$

We can observe from equation (3) that when $m_{c}=0$, then $\theta_{c}=0^{\circ}$ characterizes complex scattering from targets. Whereas, when $m_{c}=1$, then $\theta_{c}=45^{\circ}$, characterizes pure scattering from deterministic targets (i.e., trihedral or dihedral). Therefore, the pseudo scattering-type parameter $\theta_{c} \in\left[0^{\circ}, 45^{\circ}\right]$ characterizes different scattering information in between these two cases. 

between 0 to 1 .

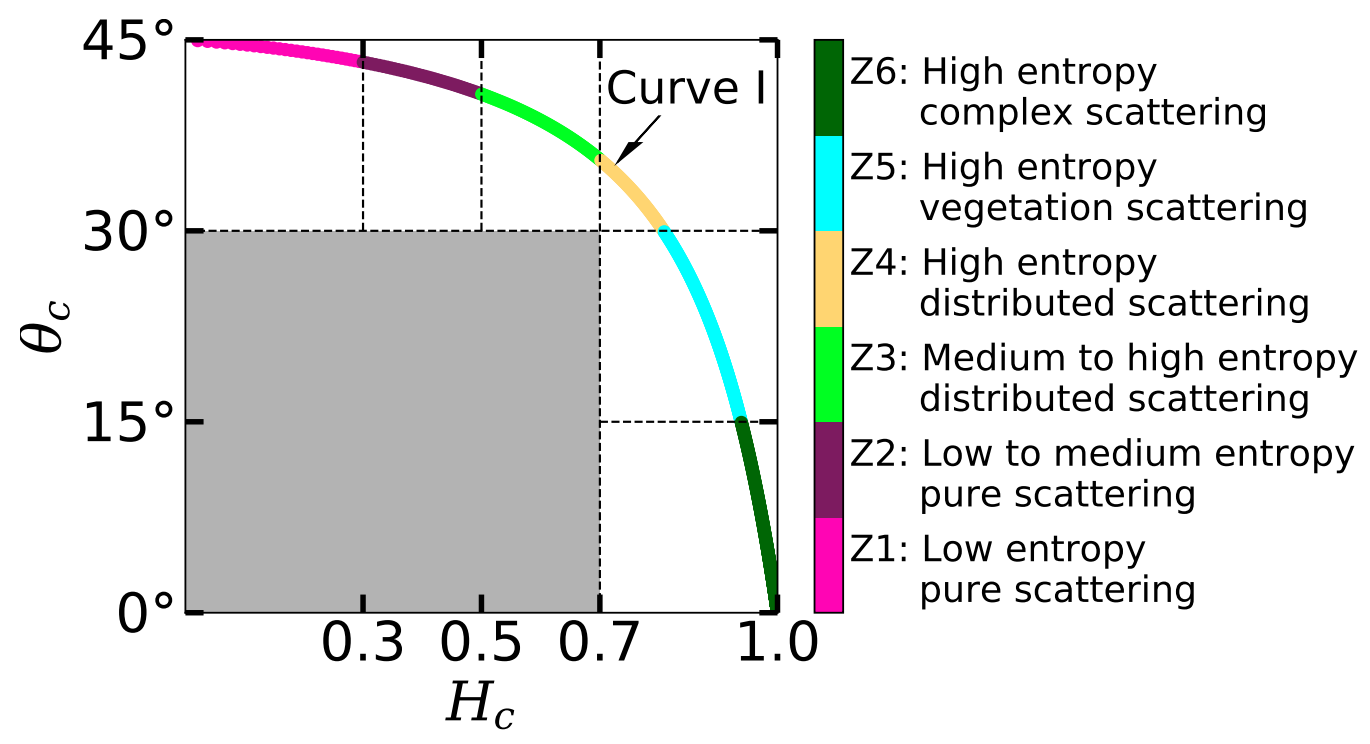

$$
H_{c}=-\sum_{i=1}^{2} p_{i} \log _{2} p_{i} ; \quad 0 \leq H_{c} \leq 1
$$

where $p_{1}=\frac{1}{1+q}$ and $p_{2}=\frac{q}{1+q}$ are the two pseudo probability measures with $p_{1} \geq p_{2}$. We can observe that $H_{c}=1$ for $p_{1}=p_{2}$ (i.e., $q=1$ ), whereas $H_{c}=0$ for $p_{1}=1$ (i.e., $\left.q=0\right)$.

Using $\theta_{c}$ and $H_{c}$ together, we propose an unsupervised clustering framework shown in Fig. 4. The curve (Curve I) represents the unique feasible clustering section in the $H_{c} / \theta_{c}$ plot. It can be noted that this curve is determined from the theoretical relationship between $\theta_{c}$ and $H_{c}$ while varying $m_{c}$

Figure 4: The $H_{c} / \theta_{c}$ 2D clustering sections. The curve is divided into six zones: $\mathrm{Z} 1$ to $\mathrm{Z} 6$.

201

Next, we define the pseudo scattering entropy parameter as,

Based on particular scattering characteristics from targets Cloude and Pottier, 1997), we propose six possible clustering zones: Z1, Z2, Z3, Z4, Z5 


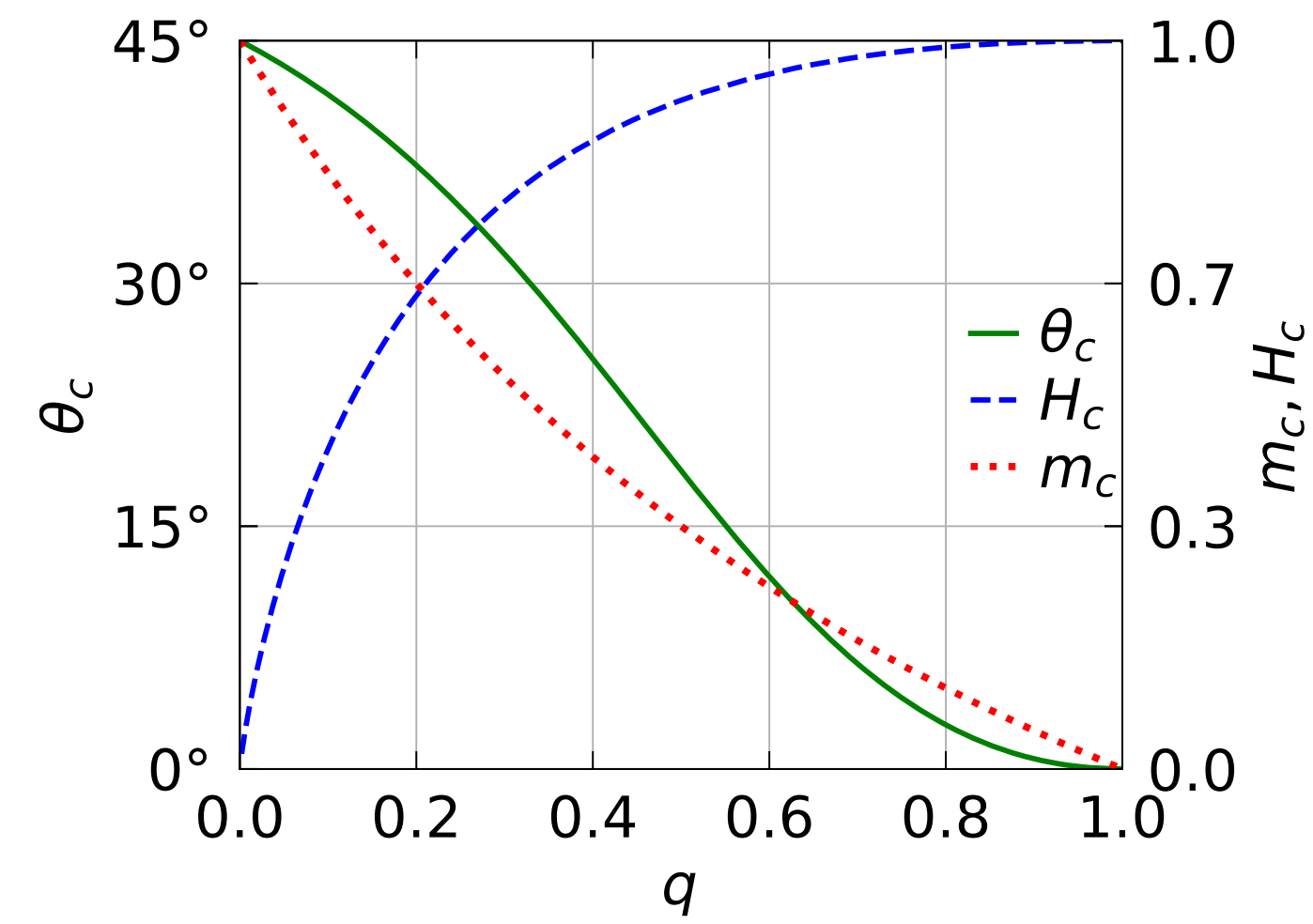

Figure 5: Relationship between the proposed descriptors $\left(\theta_{c}, m_{c}, H_{c}\right)$ and $q$.

210 and $[0.7,1.0]$, and $\theta_{c}$ into three sub-categories: $\left[0^{\circ}, 15^{\circ}\right),\left[15^{\circ}, 30^{\circ}\right),\left[30^{\circ}, 45^{\circ}\right]$.

Each of these zones represents different scattering phenomena from the scene. The relationship between $q$ and the three proposed descriptors $\left(\theta_{c}, m_{c}\right.$, and $H_{c}$ ) along with the boundary values of the clustering zones is shown in Figure 5 and Table 2 .

Cloude (2007) proposed the $H / \bar{\alpha}$ clustering technique for dual-polarimetric (HH-HV or VV-VH) SAR data. Unlike full-polarimetric measurements, the $2 \times 2$ covariance matrix is formed using only the column of the scattering matrix to characterize various targets. The scattering angle $\alpha$ is obtained from the eigenvector parameterization, and $H$ is obtained from the eigenvalues of

and Z6 by splitting $H_{c}$ into four sub-categories: $[0,0.3),[0.3,0.5),[0.5,0.7)$, 
Table 2: Boundary values of the descriptors $\left(H_{c}, \theta_{c}, q\right.$, and $\left.m_{c}\right)$ for adjacent zones in the proposed clustering framework

\begin{tabular}{ccccc}
\hline & $H_{c}$ & $\theta_{c}$ & $q$ & $m_{c}$ \\
\hline- & 0 & $45^{\circ}$ & 0 & 1 \\
$\mathbf{Z 1 - Z 2}$ & 0.30 & $43.25^{\circ}$ & 0.06 & 0.89 \\
$\mathbf{Z 2 - Z 3}$ & 0.50 & $40.74^{\circ}$ & 0.12 & 0.78 \\
$\mathbf{Z 3 - Z 4}$ & 0.70 & $35.59^{\circ}$ & 0.23 & 0.62 \\
$\mathbf{Z 4 - Z 5}$ & 0.81 & $30.00^{\circ}$ & 0.33 & 0.51 \\
$\mathbf{Z 5 - Z 6}$ & 0.94 & $15.00^{\circ}$ & 0.55 & 0.29 \\
- & 1 & $0^{\circ}$ & 1 & 0 \\
\hline
\end{tabular}

the covariance matrix as pseudo probabilities. The average scattering angle $\bar{\alpha}$ is obtained from the two orthogonal polarization states weighted with the two corresponding pseudo probabilities.

It is important to note that we cannot directly apply the $H / \bar{\alpha}$ decomposition technique to characterize target scattering mechanisms for GRD SAR data. Hence, to characterize targets utilizing GRD data, we propose an equivalent scattering angle $\theta_{c}$ based on the approach presented in (Dey et al., 2020a). We present the comparison of the two scattering angles for elementary targets and volume scattering models from a random cloud of anisotropic particles in Table 3 . For comparison purpose, the scattering angle $\theta_{c}$ is scaled to the same range of $\bar{\alpha}$ as, $\bar{\theta}_{c}=45^{\circ}-\theta_{c}$. We can note that all elementary targets reside at the origin, whereas the volume scattering models reside precisely on the lower curve of the $H / \bar{\alpha}$ plane.

Unlike the unsupervised clustering plane formed from the dual-pol $H / \alpha$ framework, the proposed $H_{c} / \theta_{c}$ framework forms a clustering segment. Both $\theta_{c}$ and $H_{c}$ are derived from the cross-pol ratio $q$. However, their physical interpretations for targets are quite different due to their fundamental formula- 
Table 3: Comparison between Cloude $\bar{\alpha}$ and $\bar{\theta}_{c}$ for elementary targets and volume scattering models for dual-polarimetric SAR data.

\begin{tabular}{cccccc}
\hline & Trihedral & Dihedral & Prolate & Oblate & Noise (Identity) \\
\hline $\bar{\alpha}$ & $0^{\circ}$ & $0^{\circ}$ & $22.5^{\circ}$ & $10^{\circ}$ & $45^{\circ}$ \\
\hline $\bar{\theta}_{c}$ & $0^{\circ}$ & $0^{\circ}$ & $15.3^{\circ}$ & $4.3^{\circ}$ & $45^{\circ}$ \\
\hline$H=H_{c}$ & 0 & 0 & 0.811 & 0.503 & 1 \\
\hline
\end{tabular}

tion, even though there is some correlation between the two parameters. On the one hand, the derivation of $H_{c}$ is equivalent to the von Neumann type of entropy (represented as Shannon entropy) utilizing the pseudo-probabilities in terms of $q$. On the other hand, the derivation of $\theta_{c}$ follows from the equivalent formalism given in (Dey et al., 2020a). It characterizes scattering-type information using co-pol purity $\left(m_{c}\right)$ and total intensity $(I)$ in terms of $q$. One can note that their combined use is also supported by a better separation of clusters when the thresholds are defined by any one of these parameters.

The division of the clustering segment is realized from the symmetry relation for the scattering of a polarized wave. The input Stokes vector $\mathbf{S}_{i}$ and output Stokes vector to the scattering medium $\mathbf{S}_{o}$ are related by a linear relation of the form: $\mathbf{S}_{o}=\mathbf{K S}_{i}$. Several restrictions are attributed to the Kennaugh matrix $\mathbf{K}$ depending upon the symmetry and reciprocity requirements. Scattering from symmetrical medium makes $\mathbf{K}$ diagonal. In the limit of weak scattering, the linear response of the scattering medium is determined by the ensemble-averaged covariance satisfying the Bethe-Salpeter equation (Cloude and Pottier, 1997). Following some rigorous computation, $\mathbf{S}_{o}$ can be expressed as a function of the number of scattering events, $n$. Having specified $\mathbf{S}_{o}$, we can formulate the expression of the degree of polarization, $m$ 
in terms of $n$. From the definition of entropy $S\left(H_{c}\right.$ in this context) given in (Brosseau, 1991; Bicout and Brosseau, 1992), which is a function of the degree of polarization $m$, satisfying the inequality: $S(m=1) \leq S \leq S(m=0)$. Therefore, we observe that $S$ increases with increase in $n$, as, $S(n=0)=0$; $S(n=1) \approx 0.3 ; S(n=2) \approx 0.5$, and, $S(n \geq 3) \approx 0.7$, and further increasing $n$ (i.e., higher-order scattering), $S$ saturates for both dual- and full-pol case. Furthermore, for dual cross-pol case, $H_{c} \approx 0.7$ for randomly oriented cylinders. A similar dependency of the scattering-type parameter (Cloude $\alpha$ ) can also be observed as a function of the order of scattering $n$. We can approximately translate this observation to $\theta_{c}$.

\subsection{Effect of system parameters on the proposed descriptors}

In this section, we show the analysis of the effect of polarization combination and frequency. Also, we present a comparative study of conventional dual-pol descriptors from SLC data and the proposed dual-pol descriptors from dual-pol GRD SAR data. In this context, we have utilized the RADARSAT-2 (C-band) and UAVSAR (L-band) data acquired over a Canadian test site for wheat. During the acquisition, wheat was at flowering to heading stage. The sampling points consists of acquisitions from two dates (29 June 2012 and 14 July 2012), and the incidence angle ranges from $22.2^{\circ}$ to $26.5^{\circ}$.

We know that longer wavelength SAR signal (L-band) penetration depth is higher than the shorter SAR signal (C-band). Moreover, a shorter wavelength SAR signal (C-band) will suffer relatively more attenuation within vegetation canopies than a longer wavelength SAR signal (L-band). Therefore, we observe the differences in the proposed descriptors for different in- 
cident frequencies. From Table 4, we can observe higher values of $m_{c}$ and lower values of the pseudo scattering entropy in the case of L-band compared to the C-band. It may be due to less attenuation of the L-band compared to C-band. Similarly, we observe that the values of $\theta_{c}$ are more towards a pure scattering-type in the L-band than the C-band in both the dual-pol combinations. Also, we observe some effects of polarization combination on the descriptors. The predominantly vertical structure of the wheat canopy leads to higher interaction of the V-pol than the H-pol. Hence, we observe higher scattering entropy $H_{c}$ and lower co-pol purity $m_{c}$ in the $\mathrm{VV}+\mathrm{VH}$ combination.

Besides, we observed higher values of the scattering-type parameter $\theta_{c}$ in the $\mathrm{HH}+\mathrm{HV}$ combination than the $\mathrm{VV}+\mathrm{VH}$ combination. The high value of $\theta_{c}$ indicates that the scattering mechanism is comparatively purer in the $\mathrm{HH}+\mathrm{HV}$ combination than the $\mathrm{VV}+\mathrm{VH}$ combination due to less interaction of H-pol with the vertically oriented crop canopy than the V-pol.

Table 4: Effect of frequency on the proposed dual-pol descriptors for wheat.

\begin{tabular}{|c|c|c|c|c|c|c|}
\hline \multirow{2}{*}{ Frequency } & \multicolumn{3}{|c|}{$\mathrm{HH}+\mathrm{HV}$} & \multicolumn{3}{|c|}{$\mathrm{VV}+\mathrm{VH}$} \\
\hline & $m_{c}$ & $H_{c}$ & $\theta_{c}$ & $m_{c}$ & $H_{c}$ & $\theta_{c}$ \\
\hline C-band $(5.405 \mathrm{GHz})$ & $0.79 \pm 0.08$ & $0.47 \pm 0.12$ & $40.78^{\circ} \pm 2.30^{\circ}$ & $0.53 \pm 0.12$ & $0.78 \pm 0.1$ & $30.33^{\circ} \pm 6.59^{\circ}$ \\
\hline L-band $(1.258 \mathrm{GHz})$ & $0.93 \pm 0.03$ & $0.22 \pm 0.07$ & $43.84^{\circ} \pm 0.59^{\circ}$ & $0.83 \pm 0.08$ & $0.4 \pm 0.14$ & $41.79^{\circ} \pm 2.15^{\circ}$ \\
\hline
\end{tabular}

A comparative study between the conventional SLC dual-pol descriptors: Barakat degree of polarization $m$, scattering entropy $H$ and Cloude $\bar{\alpha}$, and the proposed GRD dual-pol descriptors: co-pol purity $m_{c}$, pseudo scatteringentropy $H_{c}$, and pseudo scattering-type parameter $\theta_{c}$. Table 5 shows the values of the conventional dual-pol descriptors and the proposed descriptors for two dual-pol combinations for the above experiment setup. We observe 
a negligible difference between $H$ and $H_{c}$, and $m$ and $m_{c}$. However, we observe a noticeable difference between the scattering-type parameters, $\bar{\alpha}$ and $\bar{\theta}_{c}$ (kindly note that $\bar{\theta}_{c}=45^{\circ}-\theta_{c}$ ). This difference could be because of the parameterization of the eigenvector of the $\mathbf{C}_{2}$ matrix while deriving the Cloude $\alpha$. Hence, we can say that our proposed parameters obtained from dual-pol GRD SAR data possess equivalent information as the conventional parameters derived from dual-pol SLC data.

Table 5: Comparison of conventional dual-pol descriptors from SLC data and the proposed dual-pol descriptors from dual-pol GRD SAR data.

\begin{tabular}{|c|c|c|c|c|c|c|}
\hline \multirow{2}{*}{ Channels } & \multicolumn{3}{|c|}{ SLC } & \multicolumn{3}{|c|}{ GRD } \\
\hline & $m$ & $H$ & $\bar{\alpha}$ & $m_{c}$ & $H_{c}$ & $\overline{\theta_{c}}$ \\
\hline $\mathrm{HH}+\mathrm{HV}$ & $0.80 \pm 0.08$ & $0.45 \pm 0.15$ & $12.23^{\circ} \pm 4.24^{\circ}$ & $0.79 \pm 0.08$ & $0.47 \pm 0.12$ & $4.22^{\circ} \pm 2.3^{\circ}$ \\
\hline $\mathrm{VV}+\mathrm{VH}$ & $0.55 \pm 0.11$ & $0.76 \pm 0.10$ & $24.27^{\circ} \pm 5.45^{\circ}$ & $0.53 \pm 0.13$ & $0.78 \pm 0.10$ & $14.67^{\circ} \pm 6.59^{\circ}$ \\
\hline
\end{tabular}

\subsection{Sentinel-1 dual-pol descriptors in GEE}

This section describes the extraction process of the proposed polarimetric descriptors from the Sentinel-1 dual-pol GRD SAR data on the GEE platform. The overall processing framework comprises three major blocks: data preparation, clustering and temporal analysis, as shown in Figure 6.

In the data preparation block, we import the Level-1 Ground Range Detected (GRD) Sentinel-1 backscatter coefficient (i.e., $\sigma^{\circ}$ in decibel) data into the GEE platform. The imported temporal data stack is cloud filtered using three filters:

- Metadata filter (bands: VV, VH, incidence angle, instrument mode: IW, and orbit: ascending)

- Temporal filter (date range: 25 May 2016 to 17 August 2016) 

a $5 \times 5$ boxcar filter to despeckle the data.
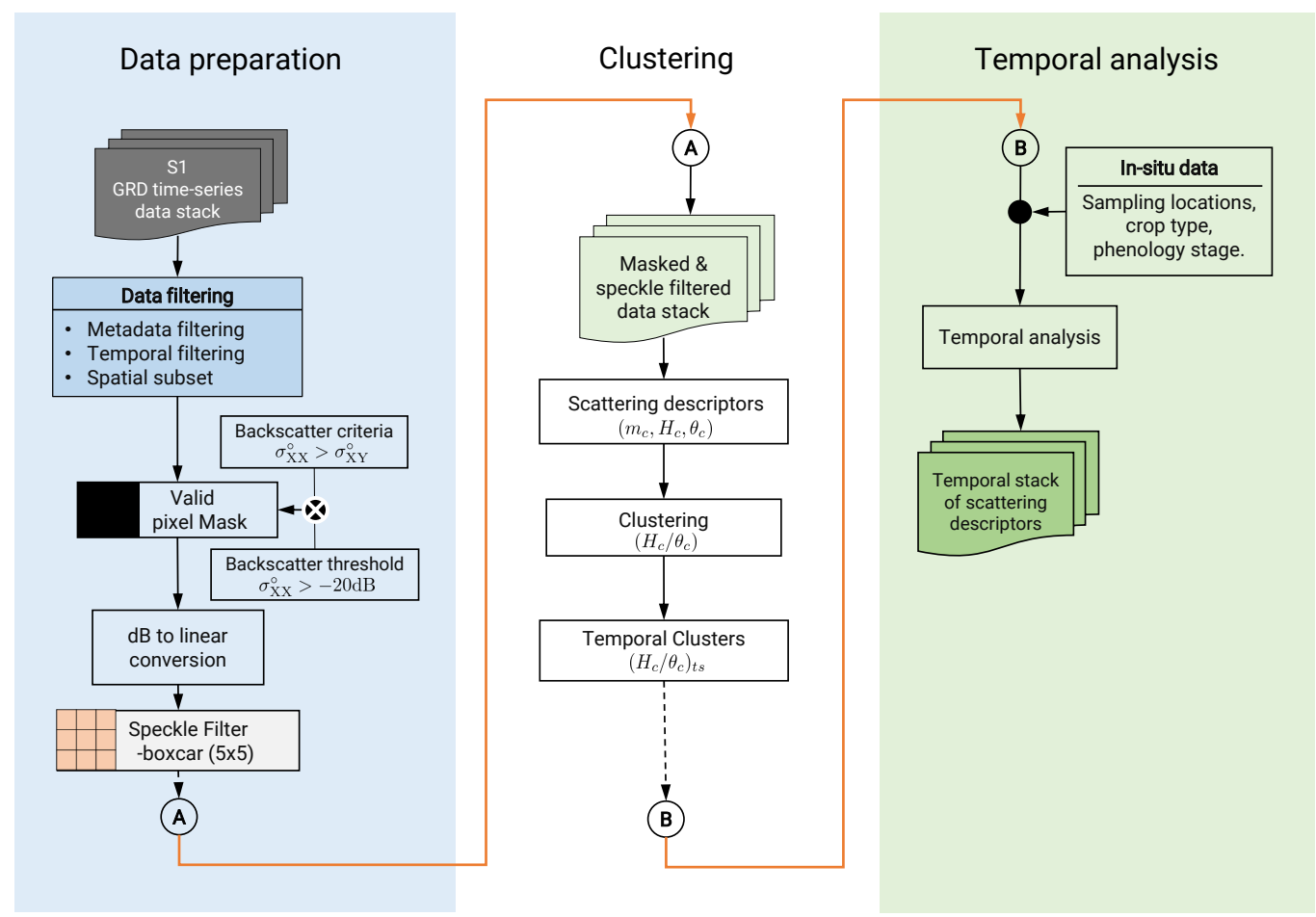

Figure 6: The proposed schematic workflow to derive the dual-polarimetric descriptors from Sentinel-1 dual-pol GRD SAR data on the GEE platform.

we utilize these descriptors to generate the $H_{c} / \theta_{c}$ clusters for each scene in 
the temporal stack. The temporal analysis block starts by importing insitu data such as sampling locations, crop type and crop phenology stages. Subsequently, we utilize these data to analyze the temporal stack of dual-pol descriptors and the $H_{c} / \theta_{c}$ clusters. Further, to complement the analysis, we generate temporal maps of each descriptor over the study area.

\section{Results and discussion}

In this section, we analyze the temporal dynamics of crops using the proposed dual-polarimetric descriptors. Furthermore, we utilize the proposed clustering framework to assess the phenological stages of the two crops (wheat, canola) from the C-band Sentinel-1 dual-pol GRD SAR data. The description of the phenological stages for wheat and canola are presented in Tables 6 and 7 respectively.

Table 6: Phenology stages of wheat. The BBCH (Biologische Bundesanstalt, Bundessortenamt und $\mathbf{C H e m i s c h e ~ I n d u s t r i e ) ~ c o d e s ~ o f ~ e a c h ~ p h e n o l o g y ~ s t a g e ~ a r e ~ a l s o ~ h i g h l i g h t e d . ~}$

\begin{tabular}{lcl}
\hline \multicolumn{1}{c}{ Phenology stage } & BBCH code & \multicolumn{1}{c}{ Description } \\
\hline Leaf development & $10-19$ & 1-9 or more leaves unfolded \\
Tillering & $20-29$ & Formation of 1-9 or more number of tillers \\
Stem elongation & $30-39$ & Elongation of first internode to fully unrolled flag leaf \\
Booting & $41-49$ & Flag leaf sheath extended to first awns visible \\
Heading & $51-59$ & First spikelet to completely emerged heads \\
Flowering-fruit development & $61-77$ & Beginning of flowering and formation of grains with milk \\
Doughstage & $83-89$ & Development of soft to Hard dough \\
Maturity & $92-97$ & Grain turns very hard and over ripened; grain loosening in day-time \\
\hline
\end{tabular}

\subsection{Temporal dynamics of the dual-polarimetric descriptors}

In this section, we present the temporal analysis of $m_{c}, \theta_{c}$ and $H_{c}$ across the phenological stages of wheat and canola over Carman, Manitoba, Canada. The spatio-temporal changes of $m_{c}, \theta_{c}$ and $H_{c}$ are shown in Figure 7 , Figure 8, and Figure 9, respectively, over the entire test site. Variations for 
Table 7: Phenology stages of canola. The BBCH (Biologische Bundesanstalt, Bundessortenamt und $\mathbf{C H e m i s c h e ~ I n d u s t r i e ) ~ c o d e s ~ o f ~ e a c h ~ p h e n o l o g y ~ s t a g e ~ a r e ~ a l s o ~ h i g h - ~}$ lighted.

\begin{tabular}{lcl}
\hline \multicolumn{1}{c}{ Phenology stage } & BBCH code & \multicolumn{1}{c}{ Description } \\
\hline Leaf development & $10-19$ & 1-9 or more leaves unfolded \\
Side shoot formation & $20-29$ & Formation of 1-9 or more side shoots \\
Stem elongation & $30-39$ & Formation of 1-9 or more extended internodes \\
Inflorescence emergence & $50-59$ & Formation of flower buds, still enclosed by leaves \\
Flowering & $60-69$ & Starting from first flower opening to the majority of petals fallen \\
Pod development & $71-79$ & Formation of pods and reaching their full size \\
Ripening & $80-89$ & Green seeds hardens and turns into dark \\
\hline
\end{tabular}

all three parameters are evident with crop growth starting from early leaf development to maturity for most agricultural fields. We also present the temporal dynamics of $H_{c} / \theta_{c}$ clusters as shown in Figure 10 , to assess the crop growth condition.

On DOY-146, most wheat and canola fields show high values of $m_{c}$ (wheat: $0.81 \pm 0.08$ and canola: $0.82 \pm 0.08$ ), $\theta_{c}$ (wheat: $41.37^{\circ} \pm 2.04^{\circ}$ and canola: $41.6^{\circ} \pm 1.91^{\circ}$ ) and medium to low value of $H_{c}$ (wheat: 0.43 \pm 0.12 and canola: $0.42 \pm 0.13)$. These responses are due to the minimal crop cover, before significant vegetative growth and leaf development. Hence, the soil characteristics (i.e., moisture and surface roughness) dominate the backscatter response. Therefore, the effect of soil roughness on the backscatter response is significant (Wiseman et al., 2014), which may have led these sample pixels to cluster in the low to medium entropy pure scattering zones (viz., Z1, Z2) in the $H_{c} / \theta_{c}$ map (Figure 10).

With crop growth advancing to the inflorescence stage, we observe an overall decrease in the values of $m_{c}$, which is evident in Figure 7 . Thus, on DOY-206, we observe medium to low values of $m_{c}$ (wheat: $0.43 \pm 0.07$ and canola: $0.42 \pm 0.1$. From the flowering to maturity stage, the canopy 

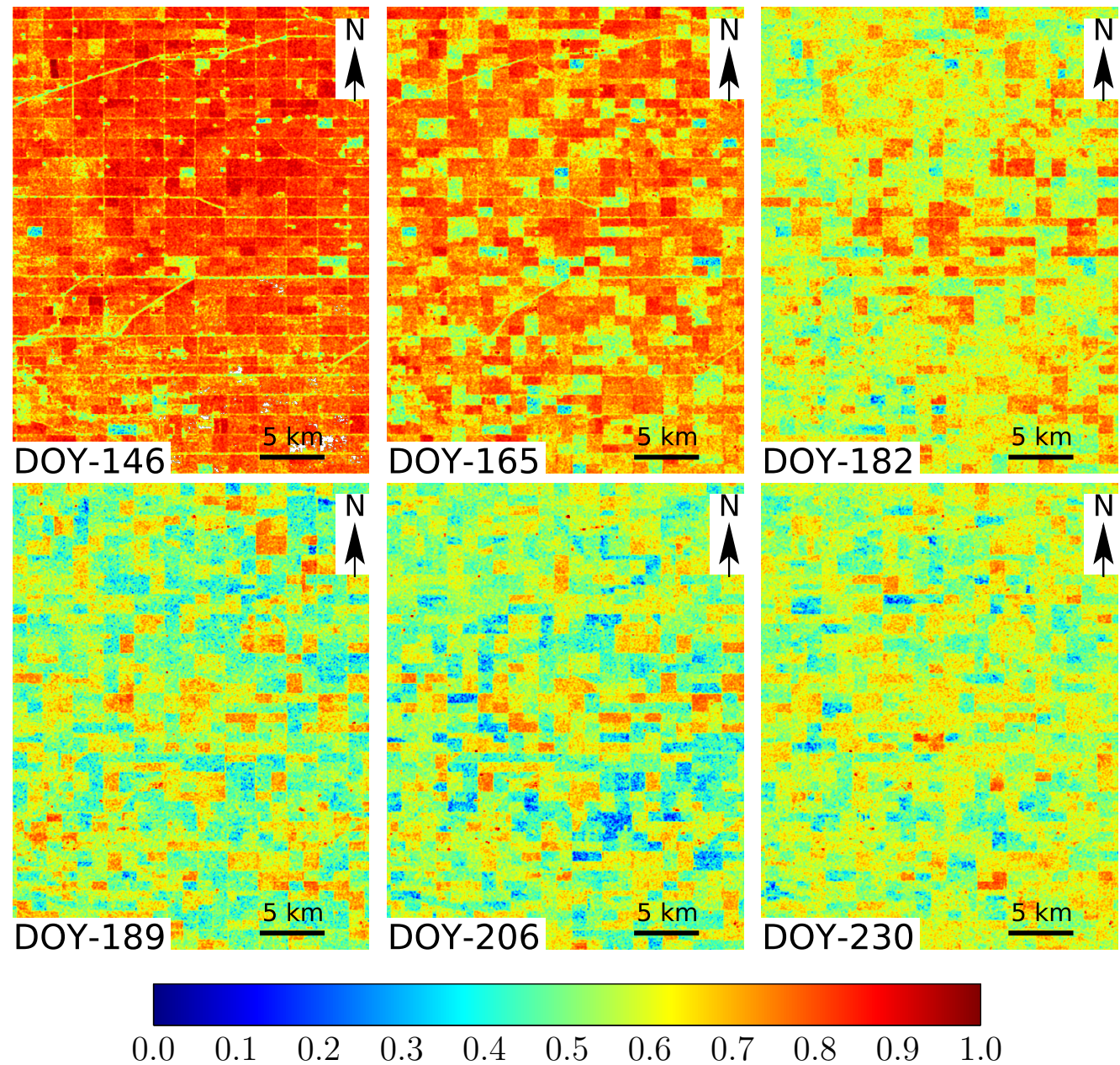

Figure 7: Temporal variation of $m_{c}$ over the study area.

361

density increases as crop biomass increases (Wiseman et al., 2014; Hariharan et al., 2018). Therefore, as reported in (Sarabandi, 1991; Wang et al., 2019; Ratha et al., 2019), we also observe similar high scattering randomness at this stage.

Moreover, during this period, the observed backscatter response is expected to be dominated by the upper canopy layer. Additionally, the values 

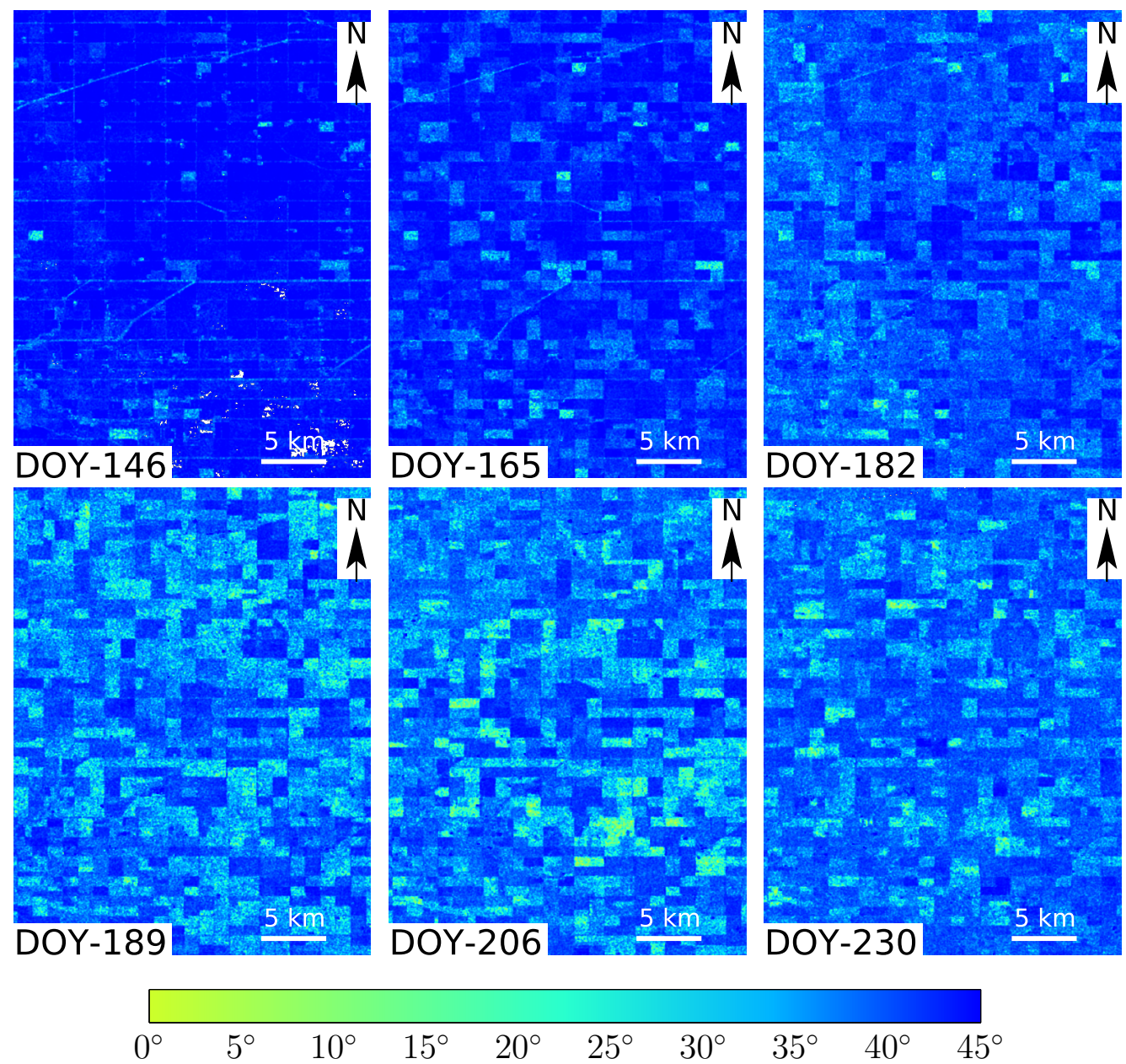

Figure 8: Temporal variation of pseudo scattering type parameter $\left(\theta_{c}\right)$ over the study area.

of $\theta_{c}$ for wheat are $24.51^{\circ} \pm 6.34^{\circ}$, and for canola $25.23^{\circ} \pm 4.7^{\circ}$ (Figure 8). These values are indicative of low random scattering within the resolution cells. We also observe an increasing trend of $H_{c}$ due to the randomly oriented canopy structure. The values of $H_{c}$ for wheat and canola are $0.86 \pm 0.06$ and $0.86 \pm 0.05$, respectively (Figure 9). Due to randomness in the vegetation structure on DOY-206, we observe dominance within the high entropy vegetation scattering zone (Z5) in Figure 10. 

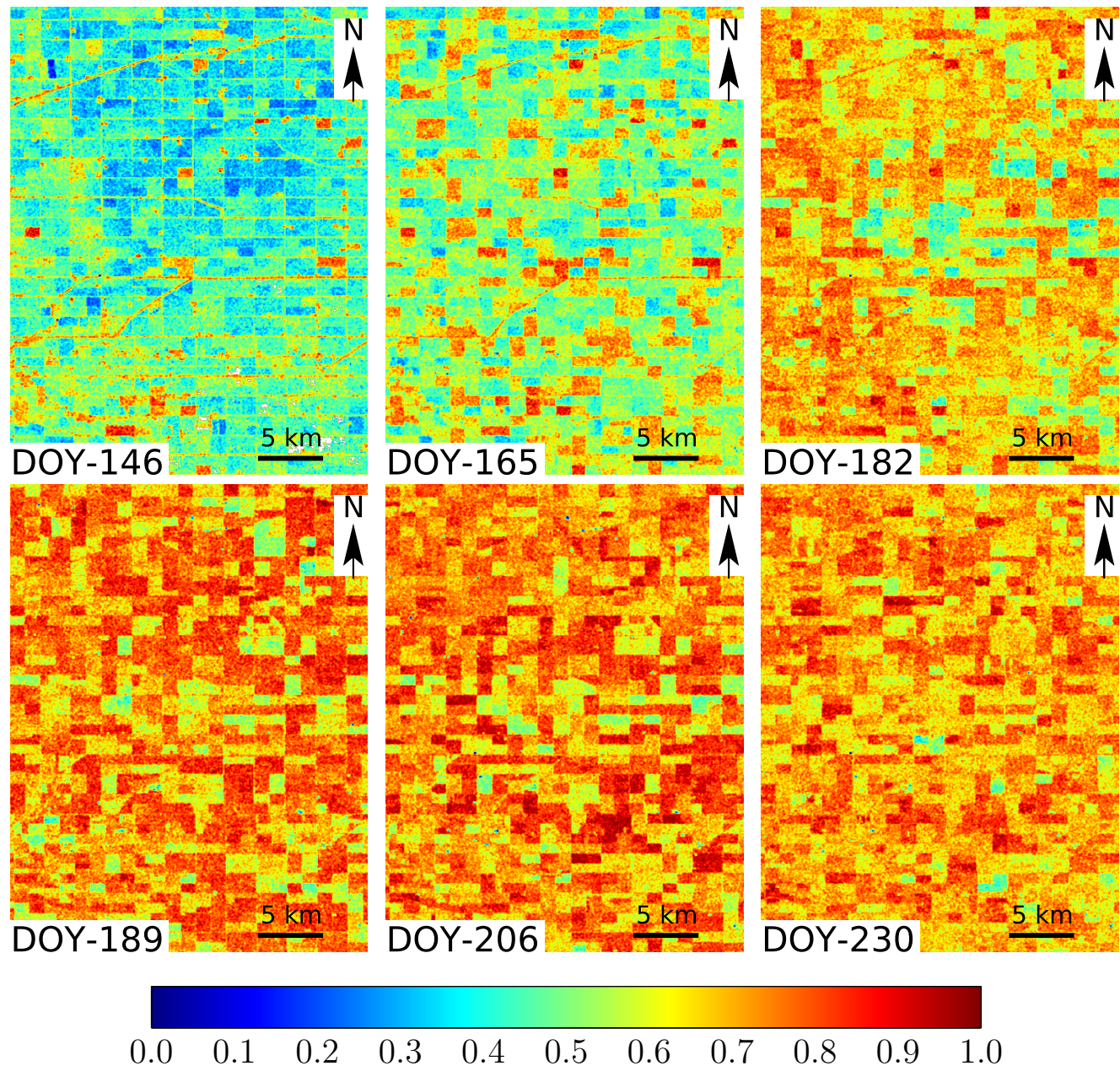

Figure 9: Temporal variation of pseudo scattering entropy parameter $\left(H_{c}\right)$ over the study area.

One can note that all three polarimetric descriptors show a trend reversal at early crop senescence. This change could be due to the randomness variation corresponding to morphology attributes with a likely decrease in canopy moisture. We can observe from Figure 7 that $m_{c}$ significantly increases during the ripening stage (DOY-230). At this stage, the values of $m_{c}$ for wheat and canola are $0.52 \pm 0.08$ and $0.55 \pm 0.08$, respectively. A similar trend is 

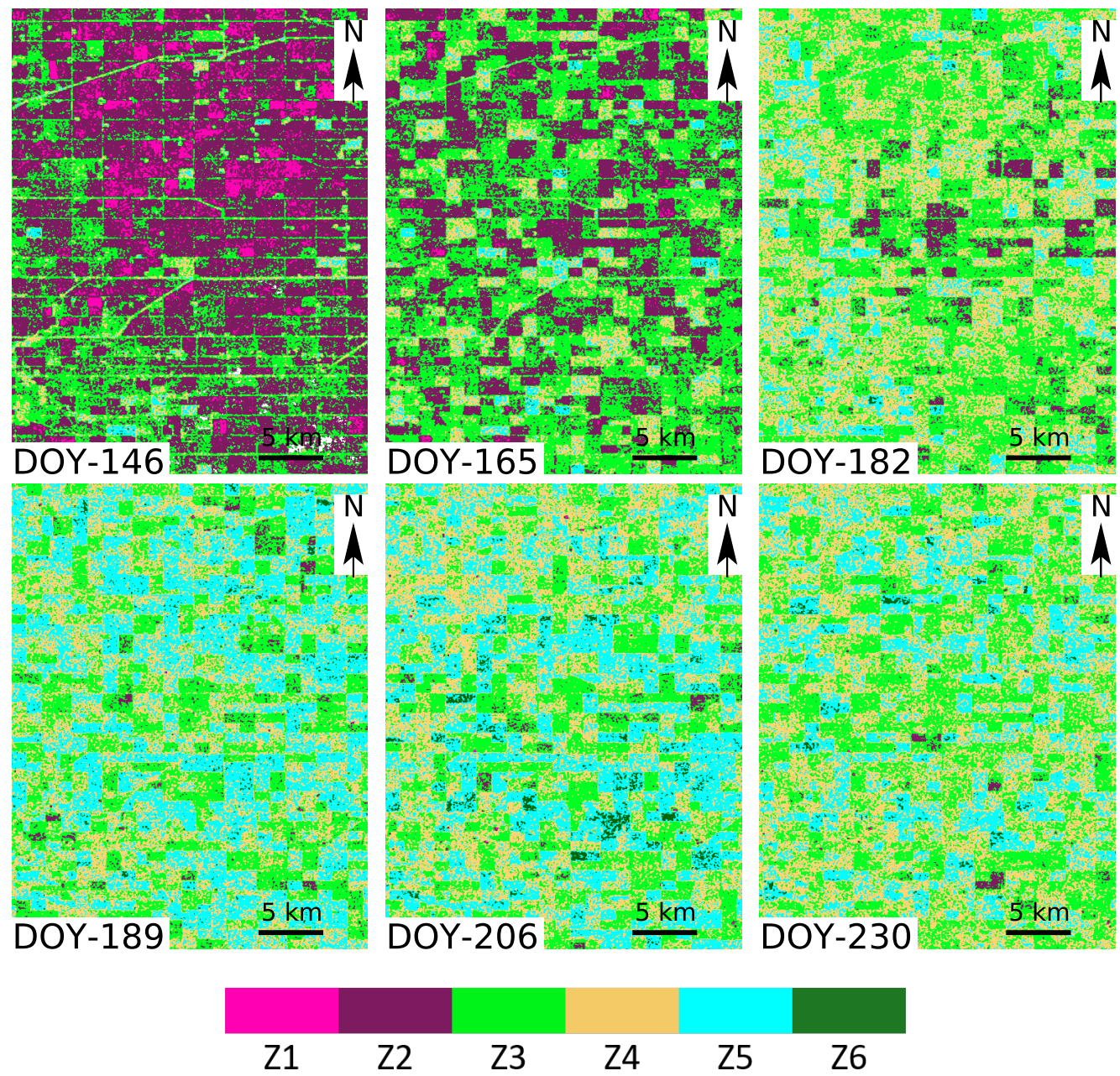

Figure 10: Temporal variation of $H_{c} / \theta_{c}$ clusters over the study area.

also observed for $\theta_{c}$ and $H_{c}$ from Figures 8 and Figure 9. These notable changes in the polarimetric descriptors might be due to the enhanced ability of the radar wave to penetrate into the moderately dry crop canopy. Also, the vegetation water content variations might have decreased the SAR signal attenuation within the resolution cell. 


\subsection{Analysis over sampling fields}

In the following sections, we provide a detailed quantitative analysis of the three descriptors $\left(m_{c}, \theta_{c}\right.$, and $\left.H_{c}\right)$ and the novel clustering framework for wheat and canola. In this study, we considered a total of 24 sample fields (wheat: 13 and canola: 11) for sensitivity and performance evaluation of the descriptors during temporal morphological changes in the canopies.

\subsubsection{Wheat}

First, we analyse temporal characteristics of $m_{c}, H_{c}$ and $\theta_{c}$ for different phenological stages of wheat. We considered a total of 48 sampling points in three different fields (Field no. 62, 220, and 233) for assessing the temporal dynamics of $\theta_{c}, H_{c}$ and $m_{c}$. We also evaluate temporal variations of the $H_{c} / \theta_{c}$ clusters according to wheat phenology.

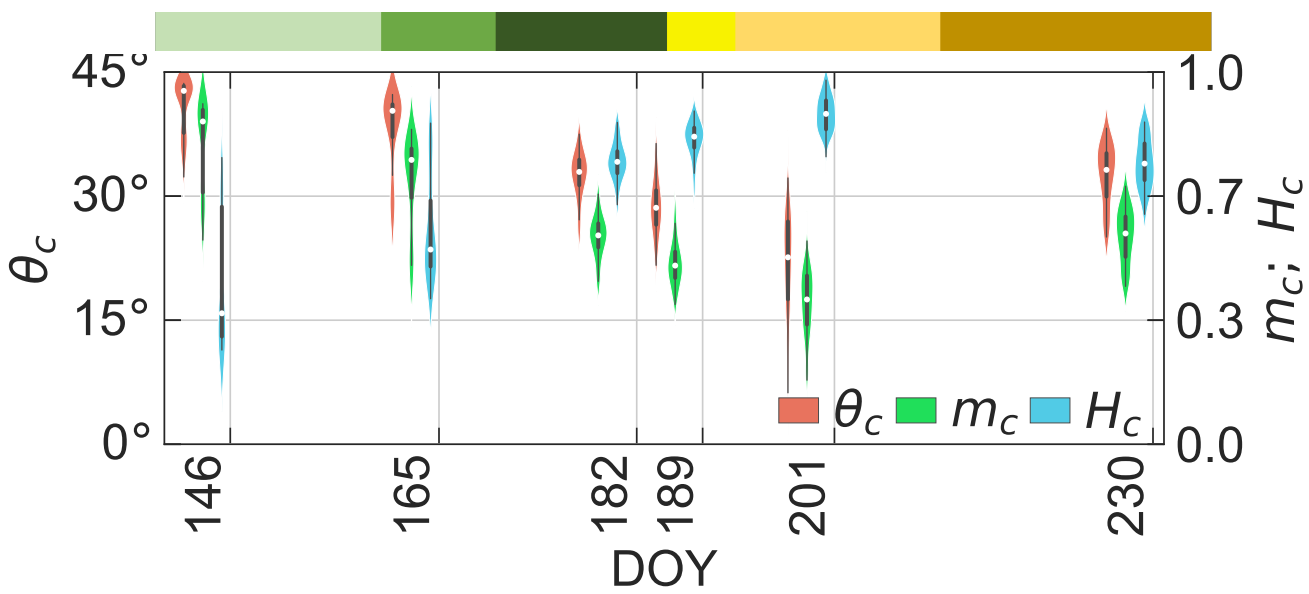

Leaf development-tillering

Stem elongation-booting

Flowering-fruit development

Dough stage

Maturity

Figure 11: Temporal variation of $m_{c}, H_{c}$ and $\theta_{c}$ for the growth stages of wheat. The white dot represents the median value, the black bar in the center represents the standard boxplot. On either side of the boxplot is a kernel density estimation displaying the shape of the data distribution. 


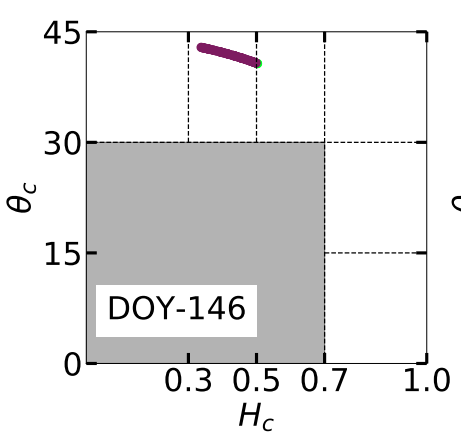

(a)

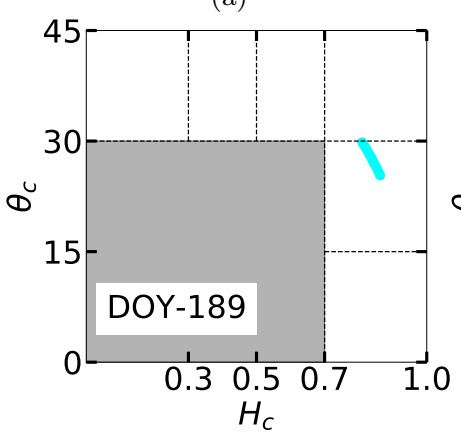

(d)

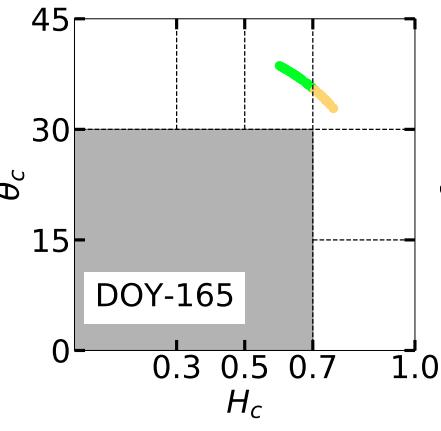

(b)

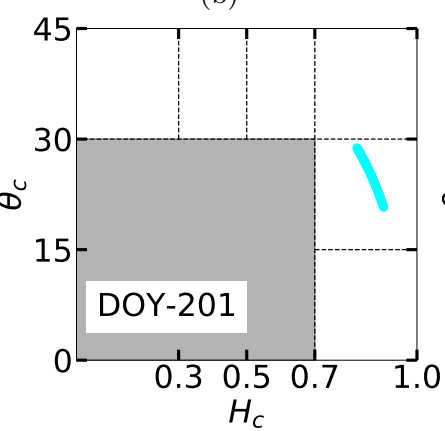

(e)

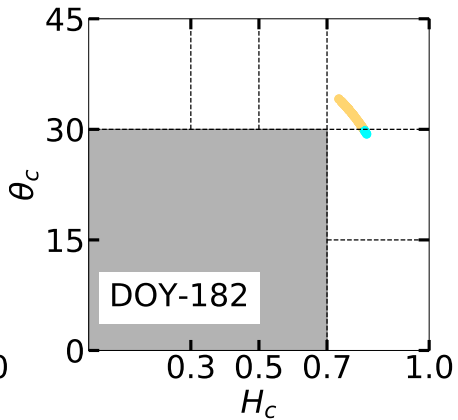

(c)

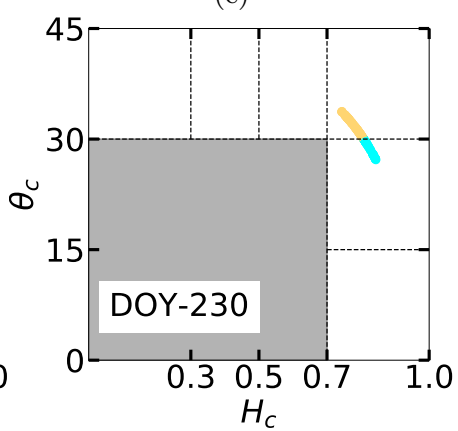

(f)

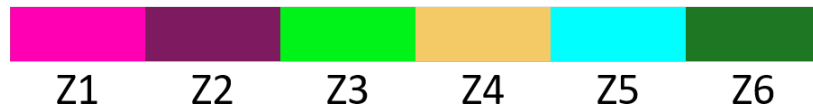

Figure 12: Temporal dynamics of the $H_{c} / \theta_{c}$ data cluster for wheat during entire growth period.

We have shown the temporal dynamics of the parameters $m_{c}, H_{c}$ and $\theta_{c}$ using a series of standard violin plots. The width of the violin represents the probability that the sampling points portion will take on the given value. We can observe from Figure 11 that $m_{c}, H_{c}$ and $\theta_{c}$ are sensitive to wheat morphological changes. For example, we note that during the early leaf development stage (around DOY-146), $m_{c}$ and $\theta_{c}$ show differential scattering information due to the presence of a minimal crop canopy. However, on DOY-201, wheat has advanced to the dough stage and consequently, the interaction of the 
radar wave with the matured canopy structure has increased. Therefore at this stage, the increase in the cross-pol component has decreased the value of $m_{c}$.

On DOY-146, high values of $m_{c}$ and $\theta_{c}$ are evident from Figure 11, reaching $0.8 \pm 0.12$ and $40.82^{\circ} \pm 3.41^{\circ}$, respectively. The distributions of $m_{c}$ and $\theta_{c}$ are left-skewed, indicating that most samples fall towards higher values of $m_{c}$ and $\theta_{c}$. These high values correspond to pure scattering, which is due to the dominance of the soil contribution relative to vegetation. As a result, most data points are clustered in the low entropy pure scattering zone (Z2) (Figure 12a). However, it is noteworthy that a fraction of the backscatter response originates from the crop leaves and side tillers. The interaction of radar waves with these structures has produced a small cluster with $\approx 11 \%$ of data points in the medium entropy zone (Z3) (Table. 8).

We note that a few fields have advanced to the tillering stage during this period due to early sowing. Hence, new tillers in those fields might have decreased $m_{c}$ and increased $H_{c}$. During stem elongation and booting, on DOY-165, we observe a decrease in $m_{c}$ and $\theta_{c}$. The values of $m_{c}$ and $\theta_{c}$ are $0.71 \pm 0.12$ and $37.87^{\circ} \pm 4.88^{\circ}$, respectively. This decrease is likely due to changes in crop morphology in the vertical direction, with an increase in the main stem and side tillers (Figure 2b). The distributions of $m_{c}$ and $\theta_{c}$ have shifted towards lower values. However, depending on the difference in the growth pattern, bi-modal distributions of $m_{c}$ and $\theta_{c}$ are observed.

During this period, high crop canopy density enhances scattering entropy, shifting the data points towards medium to high entropy zones (Z3, and Z4) as shown in Figure 12b. Hence, $57.7 \%$ of the data points are clustered within 
the medium entropy zone, while $42.3 \%$ are clustered within the high entropy zone (Table 8). Subsequently, during the heading stage (DOY-182), we observe a considerable drop in the mean values of $m_{c}$ and $\theta_{c}$ from Figure 11 . These values decrease by $21.13 \%$ and $14.21 \%$, respectively, when compared to the previous date.

On DOY-182, the crops are in their advanced vegetative stage. The radar response is similar for all fields due to their comparable scattering randomness. The standard deviations of the sample distributions have decreased significantly. During this period, we observe from Figure $12 \mathrm{c}$ a shift in the data clusters from the medium entropy (Z3) to the high entropy zone (Z4). This shift could be due to changes in the wheat canopy structure during the heading stage. At this stage, the distribution of plant biomass shifts towards the upper layer of the canopy. Thus, a major contribution of scattering is from the upper canopy layer. A small proportion of data points $(\approx 15 \%)$ are clustered in the vegetation scattering zone (Z5) (Table. 8), which might be due to early flowering of these wheat fields.

With the advancement of wheat phenology to flowering stage (Figure 2d) on DOY-189, we observe a further drop in $m_{c}(0.49 \pm 0.06)$ and $\theta_{c}\left(28.72^{\circ}\right.$ $\left.\pm 3.39^{\circ}\right)$. During this period, the wheat canopy forms a complex structure due to the appearance of flowers on the upper portion of the canopy layer. Interestingly, randomness in scattering during the flowering stage is more noticeable in the distribution of $\theta_{c}$ values. Moreover, the tail of the distribution becomes more comprehensive than the previous growth stage (Figure 11). The spread in the distribution of $\theta_{c}$ indicates multiple scattering mechanisms. Moreover, the shape of the distribution for $m_{c}$ is almost equivalent 
to $\theta_{c}$, with an overall shift towards lower values. An increase in pseudo entropy has displaced the $H_{c} / \theta_{c}$ cluster towards the high entropy vegetation scattering zone (Z5) as shown in Figure $12 \mathrm{~d}$

Dough and maturity stages continued from late July (DOY-201) to midAugust (DOY-225) with the values of $m_{c}$, and $\theta_{c}$ reaching their minimum when the crop advanced from flowering to early dough on DOY-201. The mean values of $m_{c}$ and $\theta_{c}$ reach $0.38 \pm 0.09$ and $21.56^{\circ} \pm 6.24^{\circ}$ respectively. We observe a broad spread of $\theta_{c}$ values in Figure 11, which may be due to randomly oriented wheat stems and heads (Figure 2e). Wu et al. (1985) reported a similar phenomenon given that during the heading stage, a significant portion of the total scattering occurs from the wheat heads. It is noteworthy that the distributions are bi-modal, denoting two primary scattering sources: the thick upper canopy layer and the relatively less dense bottom canopy. We observe that all data points cluster in the high entropy and vegetation scattering zone $(\mathrm{Z} 5)$ in the $H_{c} / \theta_{c}$ plot (Figure $12 \mathrm{e}$ ).

Table 8: Temporal variation in the percentage of data points in each zone for different phenology stages of wheat. The zone with the maximum number of points at a particular phenology stage is highlighted in bold. Each row represents a phenology stage, and the solid line in between two phenology stages represents a significant variation in the temporal trend for the zones.

\begin{tabular}{cccccccc}
\hline DOY & Z1 & $\mathbf{Z 2}$ & $\mathbf{Z 3}$ & $\mathbf{Z 4}$ & $\mathbf{Z 5}$ & $\mathbf{Z 6}$ & Growth stage \\
\hline 146 & 0.0 & $\mathbf{8 9 . 4}$ & 10.6 & 0.0 & 0.0 & 0.0 & Leaf Development-Tillering \\
165 & 0.0 & 0.0 & $\mathbf{5 7 . 7}$ & 42.3 & 0.0 & 0.0 & Stem Elongation-Booting \\
182 & 0.0 & 0.0 & 0.0 & $\mathbf{8 4 . 6}$ & 15.4 & 0.0 & Heading \\
\hline 189 & 0.0 & 0.0 & 0.0 & 0.0 & $\mathbf{1 0 0}$ & 0.0 & Flowering-Fruit development \\
201 & 0.0 & 0.0 & 0.0 & 0.0 & $\mathbf{1 0 0}$ & 0.0 & Dough stage \\
\hline 230 & 0.0 & 0.0 & 0.0 & $\mathbf{6 5 . 4}$ & 34.6 & 0.0 & Maturity \\
\hline
\end{tabular}


During the ripening stage, the canopy moisture content drops rapidly. As a result, penetration of the SAR signal into the crop canopy increases and hence, there is a substantial scattering contribution from the ground to the total backscatter. A trend reversal is observed for all the dual polarimetric descriptors when the crop reaches the early mature stage (DOY230). During this period, the mean values of $m_{c}$ and $\theta_{c}$ increase to $0.56 \pm$ 0.07 and $32.54^{\circ} \pm 3.65^{\circ}$, respectively. The median of the distribution shifts towards higher values (Figure 12f). We observe a decrease in the spread of distributions for both $m_{c}$ and $\theta_{c}$ in Figure 11 indicating uniformity in the scattering mechanism.

A decrease in scattering entropy shifts the $H_{c} / \theta_{c}$ cluster towards the distributed scattering zone (Z4) from random scattering, as shown in Figure $12 \mathrm{f}$. However, $34.6 \%$ of the data points are clustered within the vegetation scattering zone (Z5) with $65.4 \%$ of the data points clustered in Z4 (Table 8). The Z5 clusters appearance might be due to the late maturity of wheat, which is also in agreement with the bi-modal distribution of the $m_{c}$ and $\theta_{c}$ parameters in Figure 11 .

The proportion of data points over different scattering regions for other crop phenological stages is presented in Table 8. The results indicate a smooth transition of scattering mechanisms throughout the growing cycle of wheat. Using the proposed scattering descriptors and the novel clustering framework, we capture different scattering mechanisms at each wheat growth stage. 

the $H_{c} / \theta_{c}$ cluster according to canola phenology.

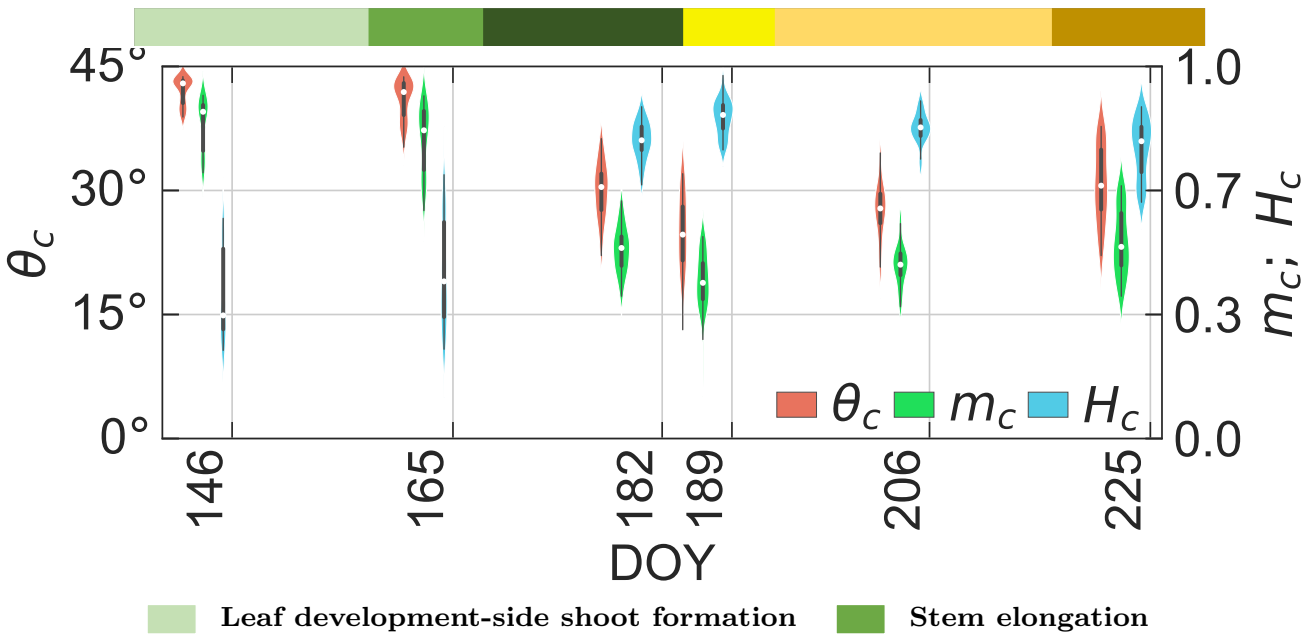

Inflorescence emergence

Flowering

Pod development

Ripening

\subsubsection{Canola}

This section analyses the temporal characteristics of $m_{c}, H_{c}$ and $\theta_{c}$ for different phenological stages of canola. In total, 48 sampling points in three canola fields (Field no. 206, 208, and 224) are used to assess the temporal dynamics of these parameters. We also evaluate the temporal variations of

Figure 13: Temporal variation of $m_{c}, H_{c}$ and $\theta_{c}$ for the growth stages of canola. The white dot represents the median value, the black bar in the center represents the standard boxplot. On either sides of boxplot is a kernel density estimation to show the distribution shape of the data.

498

Canola is a broadleaf crop with a distinctive canopy structure at every growth stage (McNairn et al., 2018; Mandal et al., 2020b). The seeding of the canola crop was completed by mid-May, as indicated in the in-situ data. Until the beginning of June, the plant advanced to its vegetative growth stage. The plant develops a dense rosette of leaves near the soil surface during the leaf development, as evident from Figure 3a. However, the size of 


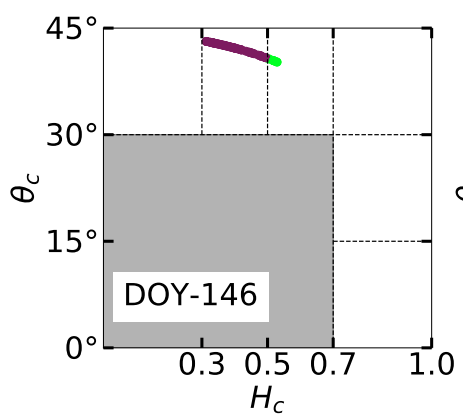

(a)

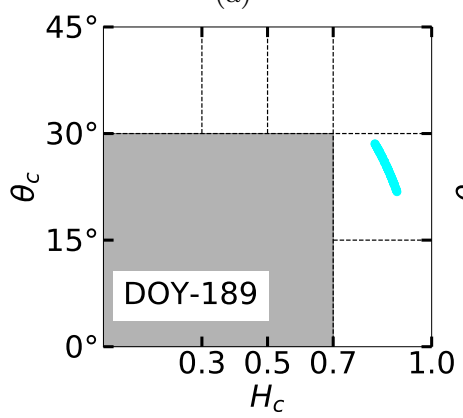

(d)

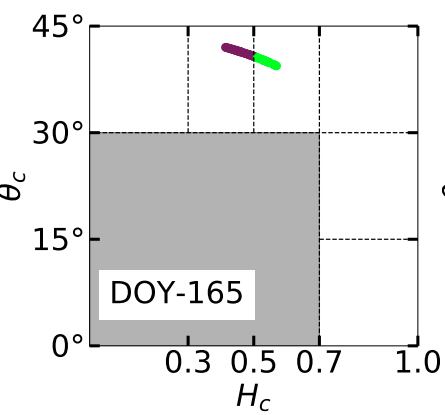

(b)

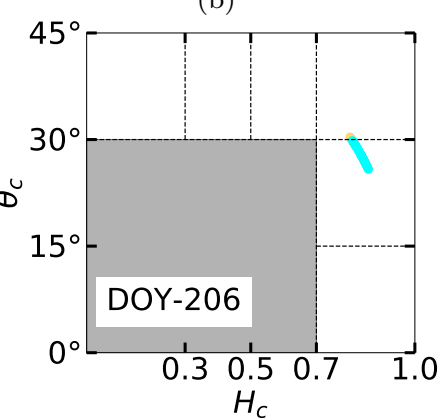

(e)

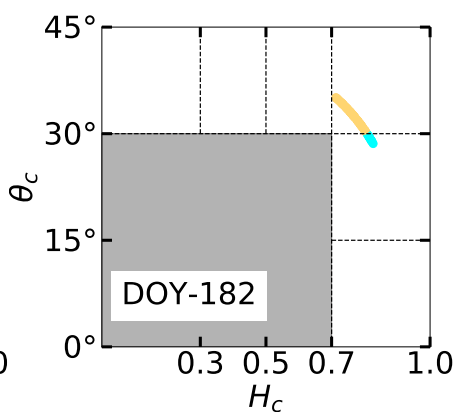

(c)

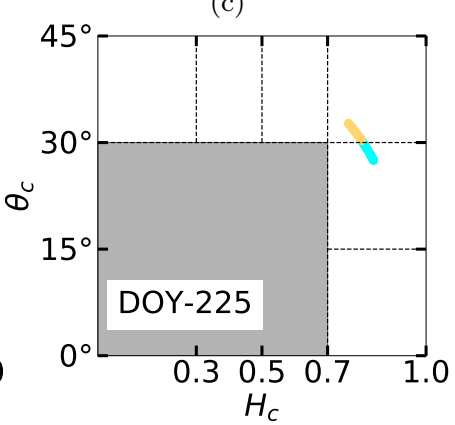

(f)

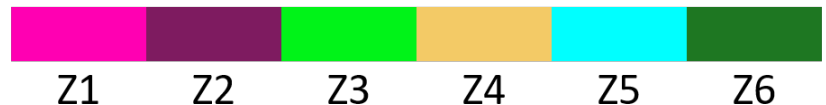

Figure 14: Temporal dynamics of the $H_{c} / \theta_{c}$ data cluster for canola during entire growth period. 
vertical plant structure can be observed from Figure 3b. At this stage, the attenuation of V-polarized waves increases. Further, an increase in biomass and PAI due to increased leaf density and branch formation leads to increased scattering randomness. On DOY-165, the $H_{c} / \theta_{c}$ cluster shifts from the pure (Z2) to the distributed scattering zone (Z3) due to a substantial amount of $m_{c}$ component during stem elongation. The accumulation of points has increased in the distributed scattering zone (Z3) due to the matured crop morphology.

During inflorescence emergence (Figure 3c), flower buds develop and leaf density increases significantly. Consequently, we observe a change in the data cluster on DOY-182 (Figure 14c). Further, the values of $m_{c}$ and $\theta_{c}$ dropped to $0.51 \pm 0.06$ and $29.94^{\circ} \pm 3.38^{\circ}$, respectively. On the other hand, the formation of branches increases scattering entropy. Hence, a shift in the $H_{c} / \theta_{c}$ cluster from medium entropy zone (Z3) to high entropy zone (Z4) is evident on DOY-182 (Figure. 14c).

During the flowering stage, the buds develop into flowers, and the main stem and branches grow (Figure 3d). On DOY-189, the scattering mechanism of all the data points is shifted towards high entropy vegetation scattering zone (Z5) (Figure 14d). We may attribute this shift to the development of a complex canopy geometry during the flowering and early pod development stage. As pods form, the canopy drops leaves. The decline of leaf cover followed by the development of pods dramatically changes the crop geometry. The canopy architecture becomes more random, with pods creating needlelike structures oriented randomly.

During the development of pods canola develops a dense, random canopy 
structure. Hence, the mean values of $m_{c}(0.47 \pm 0.06)$ and $\theta_{c}\left(27.6^{\circ} \pm 3.36^{\circ}\right)$ are minimum on DOY-206. A majority of data points $(\approx 91 \%)$ are clustered into the high entropy vegetation scattering zone (Z5). At the same time, a small percentage $(\approx 9 \%)$ of data points are clustered into Z4 (Figure $14 \mathrm{e}$ ). This small cluster may reflect the change in crop morphology as leaf area declines and the SAR signal interacts more with the needle-like canopy (Figure 3e).

Table 9: Temporal variation in the percentage of data points in each zone for different phenology stages of canola. The zone with the maximum number of points at a particular phenology stage is highlighted in bold. Each row represents a phenology stage and the solid line in between two phenology stages represents a significant variation in the temporal trend for the zones.

\begin{tabular}{cccccccl}
\hline DOY & Z1 & Z2 & Z3 & $\mathbf{Z 4}$ & $\mathbf{Z 5}$ & $\mathbf{Z 6}$ & Growth stage \\
\hline 146 & 0.0 & $\mathbf{8 9 . 8}$ & 10.2 & 0.0 & 0.0 & 0.0 & $\begin{array}{l}\text { Leaf Development } \\
\text { Side shoot formation }\end{array}$ \\
165 & 0.0 & 48.9 & $\mathbf{5 1 . 1}$ & 0.0 & 0.0 & 0.0 & Stem Elongation \\
182 & 0.0 & 0.0 & 0.0 & $\mathbf{8 4 . 1}$ & 15.9 & 0.0 & Inflorescence Emergence \\
\hline 189 & 0.0 & 0.0 & 0.0 & 0.0 & $\mathbf{1 0 0}$ & 0.0 & Flowering \\
\hline 206 & 0.0 & 0.0 & 0.0 & 9.1 & $\mathbf{9 0 . 9}$ & 0.0 & Pod Development \\
225 & 0.0 & 0.0 & 0.0 & $\mathbf{5 4 . 5}$ & 45.5 & 0.0 & Ripening \\
\hline
\end{tabular}

Subsequently on DOY-225, we observe an increase in $m_{c}$ and $\theta_{c}$ values to $0.53 \pm 0.09$ and $30.8^{\circ} \pm 4.63^{\circ}$, respectively. This increase in the values of the descriptors might be due to the decrease in overall canopy moisture content at maturity. As canopy moisture declines, the SAR signal can penetrate deeper into the crop canopy. Hence, at late maturity, there might be a greater contribution from the soil. Because of these physical changes, the $H_{c} / \theta_{c}$ 
cluster shifts towards the distributed scattering zone (Z4) (Figure 14f). The bi-modal distribution of the parameters $m_{c}$ and $\theta_{c}$ (Figure 13) indicates two major sources of scattering. In particular, some fraction of the crop may be entering the mid/end-ripening stage, resulting in higher values of $m_{c}$ and $\theta_{c}$. In contrast, other canopies may be just entering early ripening, resulting in lower values. We observe this difference in Figure 14f. Notably, $54.5 \%$ of the data points fall in distributed scattering zone (Z4), whereas $45.5 \%$ of the data points are in the vegetation scattering zone (Z5). The proportion of data points over different scattering regions for all phenological stages of canola is presented in Table 9. The results indicate a smooth transition of scattering mechanisms throughout the growing cycle. Consequently, the proposed descriptors exhibit high sensitivity to the phenological stages of both wheat and canola. Hence, these descriptors are useful in monitoring phenological changes for both crops.

\section{Conclusion}

In this study, we propose three polarimetric descriptors from dual-pol Sentinel-1 (S1) GRD SAR data. These parameter are: the pseudo scatteringtype parameter $\left(\theta_{c}\right)$, the co-pol purity parameter $\left(m_{c}\right)$, and the pseudo scattering entropy parameter $\left(H_{c}\right)$. We have expressed these descriptors in terms of $q=\sigma_{\mathrm{XY}}^{\circ} / \sigma_{\mathrm{XX}}^{\circ}$, with $0 \leq q \leq 1$. Additionally, we have proposed a novel unsupervised clustering framework using $\theta_{c}$ and $H_{c}$.

We have used the dual-pol descriptors and the novel clustering framework to analyze temporal growth dynamics of wheat and canola over a Canadian test site. The results are very encouraging in assessing crop dynamics for 
different major phenological stages. The high sensitivity of these descriptors to different crop growth stages is evident in this context.

In the scope of this study, we have characterized diverse crop phenological stages in terms of the physical scattering of the electromagnetic wave from targets using the GRD SAR data. The unsupervised clustering framework using $H_{c} / \theta_{c}$ contains six zones representing different physical scattering mechanisms. Hence, it provides essential information about the crop growth stages without any a priori knowledge and therefore very useful in interpreting the available radar data.

The temporal analysis of the proposed descriptors revealed their high sensitivity across different phenology stages of wheat and canola. The dynamic range of $\theta_{c}$ from leaf development to fruit development of wheat is $41^{\circ}$ to $21.6^{\circ}$. Similarly, the variations of $m_{c}$ and $H_{c}$ are 0.8 to 0.38 and 0.35 to 0.9 , respectively. Similar dynamic ranges of these parameters are also evident for canola crop. Furthermore, the proposed clustering schema efficiently captured the diverse phenology stage of wheat and canola. For leaf development and tillering stages of wheat, $90 \%$ of the sampling points are clustered into low to medium entropy pure scattering zone. During flowering and fruit development stages, $100 \%$ of the sampling points are shifted into high entropy vegetation scattering zone. Subsequently, during the maturity stage, the clusters of the sampling points were split between the high entropy vegetation scattering and high entropy distributed scattering zones with $>65 \%$ of the sampling points in the high entropy distributed scattering zone. Similar cluster dynamics are observed for the canola crop. However, it is computationally intensive to implement these algorithms for a high vol- 
ume of temporal data from a global agricultural monitoring perspective. To overcome this limitation, we utilized the cloud-based platform (GEE) to acquire and process the dense time-series data of Sentinel-1. Implementing the algorithms in GEE also facilitates efficient generation of global maps of crop phenology stages.

This study only used the GRD SAR data product to formulate the target characterizing descriptors demonstrating promising results for natural targets. We can further extend this study to different crop types and different dual-pol SAR sensors configurations. The proposed descriptors should be beneficial in studying natural ecosystems with upcoming dual-pol NASAISRO Synthetic Aperture Radar Mission (NISAR) and Sentinel SAR constellation.

\section{Acknowledgement}

The authors are grateful to the SMAPVEX16 science team for providing ground truth information. The authors would like to thank the Google Earth Engine team for providing the free SAR data processing platform. Authors also acknowledge the GEO-AWS Earth Observation Cloud Credits Program, which supported the computation with Sentinel-1 on AWS cloud platform through the project: "AWS4AgriSAR-Crop inventory mapping from SAR data on a cloud computing platform", and formed the testbed for processing pipelines. Mr. Narayanarao B. and Mr. Subhadip Dey would like to acknowledge the support from the Ministry of Education (formerly Ministry of Human Resource and Development-MHRD), Govt. of India, towards their doctoral research work. The authors want to thank the support of the Span- 
ish Ministry of Science and Innovation, State Research Agency (AEI) and the European Regional Development Fund under project TEC2017-85244-C2-1P. The authors are thankful to the overleaf team (https://overleaf.com/) for providing the latex editing platform.

\section{References}

Agriculture, M., B., 2016. Agriculture Province of Manitoba. URL: https://www.gov.mb.ca/agriculture/crops/seasonal-reports/ crop-report-archive/index.html.

Ainsworth, T., Kelly, J., Lee, J.S., 2009. Classification comparisons between dual-pol, compact polarimetric and quad-pol SAR imagery. ISPRS Journal of Photogrammetry and Remote Sensing 64, 464-471.

Ainsworth, T.L., Kelly, J., Lee, J.S., 2008. Polarimetric analysis of dual polarimetric SAR imagery, in: 7th European Conference on Synthetic Aperture Radar, VDE. pp. 1-4.

Ali, I., Naeimi, V., Cao, S., Elefante, S., Bauer-Marschallinger, B., Wagner, W., 2017. Sentinel-1 data cube exploitation: Tools, products, services and quality control, in: Proc. Big Data Space, pp. 40-43.

Arias, M., Campo-Bescós, M.Á., Álvarez-Mozos, J., 2020. Crop classification based on temporal signatures of sentinel-1 observations over navarre province, Spain. Remote Sensing 12, 278.

Bargiel, D., 2017. A new method for crop classification combining time 
series of radar images and crop phenology information. Remote sensing of environment 198, 369-383.

Bhuiyan, H.A., McNairn, H., Powers, J., Friesen, M., Pacheco, A., Jackson, T.J., Cosh, M.H., Colliander, A., Berg, A., Rowlandson, T., et al., 2018. Assessing SMAP soil moisture scaling and retrieval in the Carman (Canada) study site. Vadose Zone Journal 17, 1-14.

Bicout, D., Brosseau, C., 1992. Multiply scattered waves through a spatially random medium: entropy production and depolarization. Journal de Physique I 2, 2047-2063.

Bousbih, S., Zribi, M., Lili-Chabaane, Z., Baghdadi, N., El Hajj, M., Gao, Q., Mougenot, B., 2017. Potential of Sentinel-1 radar data for the assessment of soil and cereal cover parameters. Sensors 17, 2617.

Brisco, B., Brown, R., Gairns, J., Snider, B., 1992. Temporal ground-based scatterometer observations of crops in western Canada. Canadian journal of remote sensing 18, 14-21.

Brosseau, C., 1991. Polarization transfer and entropy transformation. Optik (Stuttgart) 88, 109-117.

Cloude, S., 2007. The dual polarization entropy/alpha decomposition: A palsar case study. ESASP 644, 2.

Cloude, S., 2009. Polarisation: applications in remote sensing. OUP Oxford.

Cloude, S.R., Pottier, E., 1997. An entropy based classification scheme for 
land applications of polarimetric sar. IEEE transactions on geoscience and remote sensing 35, 68-78.

Davidson, M.W., Le Toan, T., Mattia, F., Satalino, G., Manninen, T., Borgeaud, M., 2000. On the characterization of agricultural soil roughness for radar remote sensing studies. IEEE Transactions on Geoscience and Remote Sensing 38, 630-640.

De Bernardis, C.G., Vicente-Guijalba, F., Martinez-Marin, T., LopezSanchez, J.M., 2015. Estimation of key dates and stages in rice crops using dual-polarization SAR time series and a particle filtering approach. IEEE Journal of Selected Topics in Applied Earth Observations and Remote Sensing 8, 1008-1018.

Della Vecchia, A., Ferrazzoli, P., Guerriero, L., Ninivaggi, L., Strozzi, T., Wegmuller, U., 2008. Observing and modeling multifrequency scattering of maize during the whole growth cycle. IEEE Transactions on Geoscience and Remote Sensing 46, 3709-3718.

Dey, S., Bhattacharya, A., Ratha, D., Mandal, D., Frery, A.C., 2020a. Target characterization and scattering power decomposition for full and compact polarimetric SAR data. IEEE Transactions on Geoscience and Remote Sensing .

Dey, S., Bhattacharya, A., Ratha, D., Mandal, D., McNairn, H., LopezSanchez, J.M., Rao, Y., 2020b. Novel clustering schemes for full and compact polarimetric SAR data: An application for rice phenology char- 
acterization. ISPRS Journal of Photogrammetry and Remote Sensing 169, $135-151$.

ESA, 2017. Sen4cap - Sentinels for common agriculture policy. http:// esa-sen4cap.org/.

Ferrazzoli, P., Paloscia, S., Pampaloni, P., Schiavon, G., Solimini, D., Coppo, P., 1992. Sensitivity of microwave measurements to vegetation biomass and soil moisture content: A case study. IEEE Transactions on Geoscience and Remote Sensing 30, 750-756.

Fikriyah, V.N., Darvishzadeh, R., Laborte, A., Khan, N.I., Nelson, A., 2019. Discriminating transplanted and direct seeded rice using Sentinel-1 intensity data. International Journal of Applied Earth Observation and Geoinformation $76,143-153$.

Gorelick, N., Hancher, M., Dixon, M., Ilyushchenko, S., Thau, D., Moore, R., 2017. Google earth engine: Planetary-scale geospatial analysis for everyone. Remote sensing of Environment 202, 18-27.

Hariharan, S., Mandal, D., Tirodkar, S., Kumar, V., Bhattacharya, A., Lopez-Sanchez, J.M., 2018. A novel phenology based feature subset selection technique using random forest for multitemporal PolSAR crop classification. IEEE Journal of Selected Topics in Applied Earth Observations and Remote Sensing 11, 4244-4258.

Hird, J.N., DeLancey, E.R., McDermid, G.J., Kariyeva, J., 2017. Google earth engine, open-access satellite data, and machine learning in support of large-area probabilistic wetland mapping. Remote sensing 9, 1315. 
Homayouni, S., McNairn, H., Hosseini, M., Jiao, X., Powers, J., 2019. Quad and compact multitemporal C-band PolSAR observations for crop characterization and monitoring. International Journal of Applied Earth Observation and Geoinformation 74, 78-87.

Jackson, K.R., Ramakrishnan, L., Muriki, K., Canon, S., Cholia, S., Shalf, J., Wasserman, H.J., Wright, N.J., 2010. Performance analysis of high performance computing applications on the amazon web services cloud, in: 2010 IEEE second international conference on cloud computing technology and science, IEEE. pp. 159-168.

Jiao, X., Kovacs, J.M., Shang, J., McNairn, H., Walters, D., Ma, B., Geng, X., 2014. Object-oriented crop mapping and monitoring using multitemporal polarimetric RADARSAT-2 data. ISPRS Journal of Photogrammetry and Remote Sensing 96, 38-46.

Kumar, P., Prasad, R., Gupta, D., Mishra, V., Vishwakarma, A., Yadav, V., Bala, R., Choudhary, A., Avtar, R., 2018. Estimation of winter wheat crop growth parameters using time series Sentinel-1A SAR data. Geocarto international 33, 942-956.

Kussul, N., Lemoine, G., Gallego, F.J., Skakun, S.V., Lavreniuk, M., Shelestov, A.Y., 2016. Parcel-based crop classification in Ukraine using Landsat-8 data and Sentinel-1A data. IEEE Journal of Selected Topics in Applied Earth Observations and Remote Sensing 9, 2500-2508.

Lasko, K., Vadrevu, K.P., Tran, V.T., Justice, C., 2018. Mapping double and single crop paddy rice with Sentinel-1A at varying spatial scales and 
polarizations in Hanoi, Vietnam. IEEE journal of selected topics in applied earth observations and remote sensing 11, 498-512.

Lee, J.S., Grunes, M.R., Pottier, E., 2001. Quantitative comparison of classification capability: Fully polarimetric versus dual and single-polarization sar. IEEE Transactions on Geoscience and Remote Sensing 39, 2343-2351.

Li, H., Zhang, C., Zhang, S., Atkinson, P.M., 2019. Full year crop monitoring and separability assessment with fully-polarimetric L-band UAVSAR: A case study in the Sacramento Valley, California. International Journal of Applied Earth Observation and Geoinformation 74, 45-56.

Lopez-Sanchez, J.M., Cloude, S.R., Ballester-Berman, J.D., 2012. Rice phenology monitoring by means of SAR polarimetry at X-band. IEEE Transactions on Geoscience and Remote Sensing 50, 2695-2709.

Lopez-Sanchez, J.M., Vicente-Guijalba, F., Ballester-Berman, J.D., Cloude, S.R., 2014. Polarimetric response of rice fields at C-band: Analysis and phenology retrieval. IEEE Transactions on Geoscience and Remote Sensing $52,2977-2993$.

Mandal, D., Kumar, V., Bhattacharya, A., Rao, Y.S., Siqueira, P., Bera, S., 2018. Sen4Rice: A processing chain for differentiating early and late transplanted rice using time-series Sentinel-1 SAR data with Google Earth Engine. IEEE Geoscience and Remote Sensing Letters 15, 1947-1951.

Mandal, D., Kumar, V., Lopez-Sanchez, J.M., Bhattacharya, A., McNairn, H., Rao, Y., 2020a. Crop biophysical parameter retrieval from Sentinel-1 
SAR data with a multi-target inversion of Water Cloud Model. International Journal of Remote Sensing 41, 5503-5524.

Mandal, D., Kumar, V., Ratha, D., Dey, S., Bhattacharya, A., LopezSanchez, J.M., McNairn, H., Rao, Y.S., 2020b. Dual polarimetric radar vegetation index for crop growth monitoring using sentinel-1 SAR data. Remote Sensing of Environment 247, 111954.

Mascolo, L., Lopez-Sanchez, J.M., Vicente-Guijalba, F., Nunziata, F., Migliaccio, M., Mazzarella, G., 2016. A complete procedure for crop phenology estimation with PolSAR data based on the complex Wishart classifier. IEEE Transactions on Geoscience and Remote Sensing 54, 6505-6515.

McNairn, H., Brisco, B., 2004. The application of C-band polarimetric SAR for agriculture: A review. Canadian Journal of Remote Sensing 30, 525542.

McNairn, H., Jiao, X., Pacheco, A., Sinha, A., Tan, W., Li, Y., 2018. Estimating canola phenology using synthetic aperture radar. Remote Sensing of Environment 219, 196-205.

McNairn, H., Tom, J., Powers, J., Bélair, J., Berg, A., Bullock, A., Colliander, A., Cosh, A., Kim, M., Ramata, S., et al., 2016. Experimental plan SMAP validation experiment 2016 in Manitoba, Canada (SMAPVEX16MB).

Minasny, B., Shah, R.M., Che Soh, N., Arif, C., Indra Setiawan, B., et al., 2019. Automated Near-Real-Time Mapping and Monitoring of Rice Extent, Cropping Patterns, and Growth Stages in Southeast Asia Using 
Sentinel-1 Time Series on a Google Earth Engine Platform. Remote Sensing 11, 1666.

Nasrallah, A., Baghdadi, N., El Hajj, M., Darwish, T., Belhouchette, H., Faour, G., Darwich, S., Mhawej, M., 2019. Sentinel-1 data for winter wheat phenology monitoring and mapping. Remote Sensing 11, 2228.

Nelson, A., Setiyono, T., Rala, A.B., Quicho, E.D., Raviz, J.V., Abonete, P.J., Maunahan, A.A., Garcia, C.A., Bhatti, H.Z.M., Villano, L.S., et al., 2014. Towards an operational SAR-based rice monitoring system in Asia: Examples from 13 demonstration sites across Asia in the RIICE project. Remote Sensing 6, 10773-10812.

Nemani, R., Votava, P., Michaelis, A., Melton, F., Milesi, C., 2011. Collaborative supercomputing for global change science. Eos, Transactions American Geophysical Union 92, 109-110.

Nguyen, D.B., Gruber, A., Wagner, W., 2016. Mapping rice extent and cropping scheme in the Mekong Delta using Sentinel-1A data. Remote Sensing Letters 7, 1209-1218.

Periasamy, S., 2018. Significance of dual polarimetric synthetic aperture radar in biomass retrieval: An attempt on Sentinel-1. Remote Sensing of Environment 217, 537-549.

Ratha, D., Mandal, D., Kumar, V., McNairn, H., Bhattacharya, A., Frery, A.C., 2019. A generalized volume scattering model-based vegetation index from polarimetric SAR data. IEEE Geoscience and Remote Sensing Letters 16, 1791-1795. 
Redkar, T., Guidici, T., Meister, T., 2009. Windows azure platform. Springer.

Sarabandi, K., 1991. Electromagnetic scattering from vegetation canopies. .

Schlund, M., Erasmi, S., 2020. Sentinel-1 time series data for monitoring the phenology of winter wheat. Remote Sensing of Environment 246, 111814.

Singha, M., Dong, J., Zhang, G., Xiao, X., 2019. High resolution paddy rice maps in cloud-prone Bangladesh and Northeast India using Sentinel-1 data. Scientific data $6,1-10$.

Song, Y., Wang, J., 2019. Mapping winter wheat planting area and monitoring its phenology using Sentinel-1 backscatter time series. Remote Sensing 11,449 .

Steele-Dunne, S.C., McNairn, H., Monsivais-Huertero, A., Judge, J., Liu, P.W., Papathanassiou, K., 2017. Radar remote sensing of agricultural canopies: A review. IEEE Journal of Selected Topics in Applied Earth Observations and Remote Sensing 10, 2249-2273.

Trudel, M., Charbonneau, F., Leconte, R., 2012. Using RADARSAT-2 polarimetric and ENVISAT-ASAR dual-polarization data for estimating soil moisture over agricultural fields. Canadian Journal of Remote Sensing 38, $514-527$.

Ulaby, F., 1975. Radar response to vegetation. IEEE Transactions on Antennas and Propagation 23, 36-45. 
Ulaby, F.T., El-Rayes, M.A., 1987. Microwave dielectric spectrum of vegetation-Part II: Dual-dispersion model. IEEE Transactions on Geoscience and Remote Sensing, 550-557.

Van Tricht, K., Gobin, A., Gilliams, S., Piccard, I., 2018. Synergistic use of radar Sentinel-1 and optical Sentinel-2 imagery for crop mapping: a case study for Belgium. Remote Sensing 10, 1642.

Vreugdenhil, M., Wagner, W., Bauer-Marschallinger, B., Pfeil, I., Teubner, I., Rüdiger, C., Strauss, P., 2018. Sensitivity of Sentinel-1 backscatter to vegetation dynamics: An Austrian case study. Remote Sensing 10, 1396.

Wali, E., Tasumi, M., Moriyama, M., 2020. Combination of Linear Regression Lines to Understand the Response of Sentinel-1 Dual Polarization SAR Data with Crop Phenology - Case Study in Miyazaki, Japan. Remote Sensing 12, 189.

Wang, H., Magagi, R., Goïta, K., Trudel, M., McNairn, H., Powers, J., 2019. Crop phenology retrieval via polarimetric sar decomposition and random forest algorithm. Remote Sensing of Environment 231, 111234.

Whelen, T., Siqueira, P., 2018. Time-series classification of Sentinel-1 agricultural data over North Dakota. Remote sensing letters 9, 411-420.

Wiseman, G., McNairn, H., Homayouni, S., Shang, J., 2014. RADARSAT2 polarimetric SAR response to crop biomass for agricultural production monitoring. IEEE Journal of Selected Topics in Applied Earth Observations and Remote Sensing 7, 4461-4471. 
${ }_{844}$ Wu, L.k., Moore, R.K., Zoughi, R., 1985. Sources of scattering from vege845 tation canopies at 10 Ghz. IEEE Transactions on Geoscience and Remote $846 \quad$ Sensing, 737-745. 